\title{
Explicit High Order One-Step Methods for Decoupled Forward Backward Stochastic Differential Equations
}

\author{
Quan Zhou ${ }^{2,1}$ and Yabing Sun ${ }^{2, *}$ \\ ${ }^{1}$ School of Mathematics, Shandong University, Jinan, Shandong 250100, China \\ ${ }^{2}$ College of Science, National University of Defense Technology, Changsha, \\ Hunan 410073, China
}

Received 14 May 2020; Accepted (in revised version) 19 November 2020

\begin{abstract}
By using the Feynman-Kac formula and combining with Itô-Taylor expansion and finite difference approximation, we first develop an explicit third order onestep method for solving decoupled forward backward stochastic differential equations. Then based on the third order one, an explicit fourth order method is further proposed. Several numerical tests are also presented to illustrate the stability and high order accuracy of the proposed methods.
\end{abstract}

AMS subject classifications: $65 \mathrm{C} 20,65 \mathrm{C} 30,60 \mathrm{H} 35,65 \mathrm{H} 30$

Key words: Decoupled forward backward stochastic differential equations, Itô-Taylor expansion, finite difference approximation, explicit one-step method, high order convergence.

\section{Introduction}

Let $T>0$ be a deterministic terminal time and $(\Omega, \mathcal{F}, \mathbb{F}, P)$ denote a filtered complete probability space with the natural filtration $\mathbb{F}=\left(\mathcal{F}_{t}\right)_{0 \leq t \leq T}$ of an $m$-dimensional Brownian motion $W=\left(W_{t}\right)_{0 \leq t \leq T}$. Consider the decoupled forward backward stochastic differential equations (FBSDEs) on $(\Omega, \mathcal{F}, \mathbb{F}, P)$

$$
\left\{\begin{array}{l}
X_{t}=X_{0}+\int_{0}^{t} b\left(s, X_{s}\right) d s+\int_{0}^{t} \sigma\left(s, X_{s}\right) d W_{s}, \\
Y_{t}=\varphi\left(X_{T}\right)+\int_{t}^{T} f\left(s, X_{s}, Y_{s}, Z_{s}\right) d s-\int_{t}^{T} Z_{s} d W_{s},
\end{array}\right.
$$

where $t \in[0, T], X_{0} \in \mathcal{F}_{0}$ is an initial condition; $b:[0, T] \times \mathbb{R}^{d} \rightarrow \mathbb{R}^{d}$ and $\sigma:[0, T] \times \mathbb{R}^{d} \rightarrow \mathbb{R}^{d \times m}$ are, respectively, the drift and diffusion coefficients of stochastic differential equations

*Corresponding author.

Emails: quanzhoujune@163.com (Q. Zhou), sunybly@163.com (Y. Sun) 
(SDEs); $\varphi: \mathbb{R}^{d} \rightarrow \mathbb{R}^{n}$ is the terminal condition and $f:[0, T] \times \mathbb{R}^{d} \times \mathbb{R}^{n} \times \mathbb{R}^{n \times m} \rightarrow \mathbb{R}^{n}$ is the generator of backward stochastic differential equations (BSDEs). A triple $\left(X_{t}, Y_{t}, Z_{t}\right)$ is called an $L^{2}$-adapted solution of the FBSDE (1.1) if it is $\mathcal{F}_{t}$-adapted, square integrable and satisfies (1.1).

Pardoux and Peng [22] first proved the existence and uniqueness of the adapted solution of the nonlinear BSDEs. Then by using the solutions of BSDEs, Peng [24] gave a probabilistic interpretation for quasilinear parabolic partial differential equations (PDEs). Since then, the study on FBSDEs has been extensively conducted due to its applications in research on PDEs $[6,17,24]$, mathematical finance $[11,15,20]$, stochastic optimal control [14, 23], and mean-field BSDEs [2, 3, 26, 28], to name a few. However, the analytic solutions of FBSDEs are seldom known. Hence, it is important and popular to solve FBSDEs with some numerical methods.

Up to now, there have been a considerable number of numerical methods for solving BSDEs $[4,5,12,13,31-33,36,37]$ and decoupled FBSDEs [7-10,18, 19,25, 29,30,34,35,38,39] in literatures. Nevertheless, except some of the multistep methods $[30,34,37,39]$, few of one-step methods can achieve high order convergence exceeding two. In [31], the authors constructed a class of third-order one-step methods for solving BSDEs whose driver $f$ is independent of $Z$. This is the first attempt to study the third order one-step method for solving BSDEs. However, this one-step method can only solve BSDEs with $f$ not taking $Z$ as input, which causes significant limitations in application.

In this paper, we aim to design two high order one-step methods for solving general decoupled FBSDEs by extending the method given in [31]. Based on the Feynman-Kac formula, by combining with the Itô-Taylor expansions and the high order finite difference approximations, we first develop an explicit third order one-step method containing a parameter $\theta$ for FBSDEs. Then by taking $\theta=\frac{1}{2}$ and utilizing the prediction-correction method, we further propose a fourth order one-step method for FBSDEs. To attest the stability and high order accuracy of the proposed methods, we carry out some numerical experiments. All of the numerical results show that both methods are stable and high order accurate, and the fourth order method can achieve a fourth order accuracy when the weak order 4.0 Itô-Taylor scheme is used to solve SDEs.

The rest of the paper is organized as follows. In Section 2, some preliminaries on the Feynman-Kac formula, Itô-Taylor expansion, and finite difference approximation are presented, then based on which we propose an explicit third order one-step method and an explicit fourth order one-step method for decoupled FBSDEs in Section 3. In Section 4, some numerical tests are presented to show the stability and high order accuracy of the proposed methods. Finally, we give some conclusions in Section 5.

\section{Preliminaries}

\subsection{The Feynman-Kac formula}


Let $\left(X_{s}^{t, x}, Y_{s}^{t, x}, Z_{s}^{t, x}\right)$ denote the solution of the FBSDE (1.1) with the forward SDE starting from the time-space point $(t, x)$, i.e.,

$$
\left\{\begin{array}{l}
X_{s}^{t, x}=x+\int_{t}^{s} b\left(r, X_{r}^{t, x}\right) d r+\int_{t}^{s} \sigma\left(r, X_{r}^{t, x}\right) d W_{r} \\
Y_{s}^{t, x}=\varphi\left(X_{T}^{t, x}\right)+\int_{s}^{T} f\left(r, X_{r}^{t, x}, Y_{r}^{t, x}, Z_{r}^{t, x}\right) d r-\int_{s}^{T} Z_{r}^{t, x} d W_{r},
\end{array}\right.
$$

where the superscript ${ }^{t, x}$ shows that the forward SDE starts at point $(t, x)$. If no confusion is possible, this superscript is omitted.

Let $C_{b}^{l, k, k}$ denote the set of functions $\phi(t, x, y)$, which have uniformly bounded partial derivatives $\partial_{t}^{l_{1}} \phi$ and $\partial_{x}^{k_{1}} \partial_{y}^{k_{2}} \phi$ for $l_{1} \leq l$ and $k_{1}+k_{2} \leq k$. Now we recall the following Feynman-Kac formula.

Lemma 2.1 (Feynman-Kac formula). Let $f$ be continuous with respect to $s$ and uniformly Lipschitz continuous with respect to $(x, y, z)$. Assume $\varphi \in C_{b}^{2+\alpha}$ for some $\alpha \in(0,1)$. Then the solution $\left(Y_{s}^{t, x}, Z_{s}^{t, x}\right)$ of the BSDE in (2.1) can be represented as:

$$
Y_{s}^{t, x}=u\left(s, X_{s}^{t, x}\right), \quad Z_{s}^{t, x}=\left(u_{x}^{\prime} \sigma\right)\left(s, X_{s}^{t, x}\right)
$$

where $u(t, x)$ is the smooth solution of the following PDE

$$
u_{t}^{\prime}(t, x)+\left(u_{x}^{\prime} b\right)(t, x)+\frac{1}{2} \sum_{i, j=1}^{d} \sum_{k=1}^{m}\left(u_{x_{i} x_{j}}^{\prime \prime} \sigma_{i k} \sigma_{j k}\right)(t, x)+f\left(t, x, u(t, x),\left(u_{x}^{\prime} \sigma\right)(t, x)\right)=0
$$

with the terminal condition $u(T, x)=\varphi(x)$, where

$$
u_{x}^{\prime}(t, x)=\left(\begin{array}{cccc}
\frac{\partial u_{1}}{\partial x_{1}} & \frac{\partial u_{1}}{\partial x_{2}} & \cdots & \frac{\partial u_{1}}{\partial x_{d}} \\
\frac{\partial u_{2}}{\partial x_{1}} & \frac{\partial u_{2}}{\partial x_{2}} & \cdots & \frac{\partial u_{2}}{\partial x_{d}} \\
\vdots & \vdots & \ddots & \vdots \\
\frac{\partial u_{p}}{\partial x_{1}} & \frac{\partial u_{p}}{\partial x_{2}} & \cdots & \frac{\partial \varphi_{p}}{\partial x_{d}}
\end{array}\right), \quad u_{t}^{\prime}(t, x)=\left(\begin{array}{c}
\frac{\partial u_{1}}{\partial t} \\
\frac{\partial u_{2}}{\partial t} \\
\vdots \\
\frac{\partial u_{p}}{\partial t}
\end{array}\right), \quad u_{x_{i} x_{j}}^{\prime \prime}(t, x)=\left(\begin{array}{c}
\frac{\partial^{2} u_{1}}{\partial x_{i} \partial x_{j}} \\
\frac{\partial^{2} u_{2}}{\partial x_{i} \partial x_{j}} \\
\vdots \\
\frac{\partial^{2} u_{p}}{\partial x_{i} \partial x_{j}}
\end{array}\right)
$$

Remark 2.1. For the solution of the PDE (2.3), we also have the following conclusion [12]: If $b, \sigma \in C_{b}^{1+k, 3+2 k}, f \in C_{b}^{1+k, 3+2 k, 3+2 k, 3+2 k}$ and $\varphi \in C_{b}^{3+2 k+\alpha}$ for some $\alpha \in(0,1)$, then we have

$$
u \in C_{b}^{1+k, 2+2 k}, \quad k=0,1, \cdots
$$




\subsection{The Itô-Taylor expansion}

In this subsection, we recall the Itô-Taylor expansion which plays a fundamental role in constructing our high order one-step schemes. For simplicity, we consider $d=m=1$. To proceed, we introduce some definitions first.

i) Multi-index. Let $\alpha:=\left(j_{1}, j_{2}, \cdots, j_{l}\right)$ be a multi-index with $j_{i} \in\{0,1, \cdots, m\}, i=1,2, \cdots, l$. Let $l(\alpha)=l$ be the length of $\alpha$, and $n(\alpha)$ be the number of the components of $\alpha$ which are equal to zero. Denote by $v$ the multi-index of length zero, i.e., $l(v)=0$. Let $\mathcal{M}$ be the set of all multi-indices, i.e.,

$$
\mathcal{M}=\left\{\left(j_{1}, j_{2}, \cdots, j_{l}\right): j_{i} \in\{0,1, \cdots, m\}, i \in\{1, \cdots, l\}, l=1,2,3, \cdots\right\} \cup\{v\} .
$$

Given $\alpha \in \mathcal{M}$ with $l(\alpha) \geq 1,-\alpha$ and $\alpha$ - are two multi-indices obtained by deleting the first and the last component of $\alpha$, respectively. In particularly, we let $\alpha^{+}$be the multiple index after removing all the zero elements from $\alpha$, and denote

$$
\alpha=(\underbrace{0,0, \cdots, 0}_{p})
$$

by $\alpha=(0)_{p}$.

ii) Multiple Itô integrals. The multiple Itô integral operator $I_{\alpha}$ on adapted right continuous stochastic processes $\{f=f(t), t \geq 0\}$ with left limits, is defined by

$$
I_{\alpha}[f(\cdot)]_{\rho, \tau}:= \begin{cases}f(\tau), & l=0, \\ \int_{\rho}^{\tau} I_{\alpha-}[f(\cdot)]_{\rho, s} d s, & l \geq 1 \text { and } j_{l}=0, \\ \int_{\rho}^{\tau} I_{\alpha-}[f(\cdot)]_{\rho, s} d W_{s}^{j_{l}}, & l \geq 1 \text { and } j_{l} \geq 1,\end{cases}
$$

where $\rho$ and $\tau$ are two stopping times satisfying $0 \leq \rho(w) \leq \tau(w) \leq T$, a.s.. In particular, $I_{\alpha}[1]_{0, \tau}$ will be written as $I_{\alpha, \tau}$, and $I_{\alpha}[1]_{\rho, \tau}$ as $I_{\alpha, \rho, \tau}$.

iii) Itô coefficient functions. For each index $\alpha=\left(j_{1}, \cdots, j_{l}\right)$ and a function $f \in C^{r}\left(\mathbb{R}^{+} \times \mathbb{R}^{d}\right)$ with $r=l(\alpha)+n(\alpha)$, the coefficient function $f_{\alpha}$ is defined by

$$
f_{\alpha}= \begin{cases}f, & l=0, \\ L^{j_{1}} f_{-\alpha}, & l \geq 1,\end{cases}
$$

where the operators $L^{j}, j=0,1, \cdots, m$ are defined as

$$
\begin{aligned}
& L^{0} f(t, x)=\frac{\partial f}{\partial t}(t, x)+\sum_{i} b_{i}(t, x) \frac{\partial f}{\partial x_{i}}(t, x)+\frac{1}{2} \sum_{i, j}\left(\sigma \sigma^{\top}\right)_{i, j}(t, x) \frac{\partial^{2} f}{\partial x_{i} \partial x_{j}}(t, x), \\
& L^{j} f(t, x)=\sum_{k=1}^{d} \frac{\partial f}{\partial x^{k}}(t, x) \sigma_{k j}(t, x), \quad j=1, \cdots, m .
\end{aligned}
$$


iv) Hierarchical and remainder sets. We call a subset $\mathcal{A} \subset \mathcal{M}$ a hierarchical set if

$$
\mathcal{A} \neq \varnothing, \quad \sup _{\alpha \in \mathcal{A}} l(\alpha)<\infty \quad \text { and } \quad-\alpha \in \mathcal{A} \text { for each } \alpha \in \mathcal{A} \backslash\{v\},
$$

and its remainder set $\mathcal{B}(\mathcal{A})$ is defined by

$$
\mathcal{B}(\mathcal{A})=\{\alpha \in \mathcal{M} \backslash \mathcal{A}:-\alpha \in \mathcal{A}\} .
$$

With the above notations, we now state the Itô-Taylor expansion in the following lemma (see Chapter 5 in [16]).

Lemma 2.2 (Itô-Taylor expansion). Let $\rho$ and $\tau$ be two stopping times with $0 \leq \rho(w) \leq \tau(w) \leq$ $T$, and $\mathcal{A} \subset \mathcal{M}$ be a hierarchical set. For a function $f: \mathbb{R}^{+} \times \mathbb{R}^{d} \rightarrow \mathbb{R}$, the following Itô-Taylor expansion holds:

$$
f\left(\tau, X_{\tau}\right)=\sum_{\alpha \in \mathcal{A}} f_{\alpha}\left(\rho, X_{\rho}\right) I_{\alpha, \rho, \tau}+\sum_{\beta \in \mathcal{B}(\mathcal{A})} I_{\beta}\left[f_{\beta}(\cdot, X .)\right]_{\rho, \tau}
$$

providing that all of the derivatives of $b, \sigma$ and $f$ and all of the multiple Ito integrals appearing in (2.5) exist.

Moreover, for multiple stochastic integrals, the following lemma holds [16].

Lemma 2.3. If $\alpha \in \mathcal{M} \backslash\{\varnothing\}, l(\alpha) \neq n(\alpha)$, and $t_{0} \leq \rho \leq \tau \leq T<\infty$, then

$$
\mathbb{E}\left[I_{\alpha}[f(\cdot)]_{\rho, \tau} \mid \mathcal{F}_{\rho}\right]=0, \quad \text { w.p.1, }
$$

provided that the stochastic integral $I_{\alpha}[f(\cdot)]_{\rho, \tau}$ exists.

For multiple index $\alpha=\left(j_{1}, j_{2}, \ldots, j_{l}\right) \in \mathcal{M}$, let $L^{\alpha}=L^{j_{1}} \circ L^{j_{2}} \circ \cdots \circ L^{j_{l}}$, and define

$$
\begin{aligned}
& \mathfrak{g}^{\alpha}:=\left\{u:[0, T] \times \mathbb{R} \rightarrow \mathbb{R} \mid L^{\alpha} u \text { exists and is continous }\right\}, \\
& \mathfrak{g}_{b}^{\alpha}:=\left\{u:[0, T] \times \mathbb{R} \rightarrow \mathbb{R} \mid u \in \mathfrak{g}^{\alpha} \text { and } L^{\alpha} u \text { is bounded }\right\} .
\end{aligned}
$$

Denote $u\left(t, X_{t}\right)$ and $u_{t}^{\alpha}$ by, respectively, $u_{t}$ and $L^{\alpha} u_{t}$. Let $r$ be a given small positive number, then we can derive the following theorem $[16,31]$.

Theorem 2.1. If $p \geq 0$ and $u \in \mathfrak{g}_{b}^{(0)_{p+1}}$, then it holds that

$$
\mathbb{E}\left[u\left(t+r, X_{t+r}\right) \mid \mathcal{F}_{t}\right]=u_{t}+r u_{t}^{(0)}+\frac{r^{2}}{2 !} u_{t}^{(0,0)}+\cdots+\frac{r^{p}}{p !} u_{t}^{(0)_{p}}+\mathcal{O}\left(r^{p+1}\right) .
$$

Proof. By using Lemma 2.3, it is easy to deduce (2.6). For more details, please refer to [31]. We omit it here. 


\subsection{Finite difference approximations}

In this subsection, we recall some high order finite difference approximations, which play a key role in proposing our high order one-step schemes. tors

For a given function $g=g(x)$, we define the following two difference quotient opera-

$$
\begin{aligned}
& D_{h}^{0} g(x)=\frac{-g(x+2 h)+8 g(x+h)-8 g(x-h)+g(x-2 h)}{12 h}, \\
& D_{h}^{2} g(x)=\frac{-g(x+2 h)+16 g(x+h)-30 g(x)+16 g(x-h)-g(x-2 h)}{12 h^{2}},
\end{aligned}
$$

with a positive real number $h$. Using Taylor expansion, it is easy to deduce

$$
\begin{array}{ll}
D_{h}^{0} g(x)-g^{\prime}(x)=\mathcal{O}\left(h^{4}\right), & g \in C_{b,}^{5} \\
D_{h}^{2} g(x)-g^{\prime \prime}(x)=\mathcal{O}\left(h^{4}\right), & g \in C_{b}^{6} .
\end{array}
$$

We take $D_{h}^{0}$ for instance. Let $g \in C_{b}^{5}$, then by Taylor expansions

$$
\begin{aligned}
& g(x+h)=g(x)+h g^{\prime}(x)+\frac{h^{2}}{2} g^{\prime \prime}(x)+\frac{h^{3}}{3 !} g^{\prime \prime \prime}(x)+\frac{h^{4}}{4 !} g^{(4)}(x)+\frac{h^{5}}{5 !} g^{(5)}\left(\xi_{1}\right), \\
& g(x-h)=g(x)-h g^{\prime}(x)+\frac{h^{2}}{2} g^{\prime \prime}(x)-\frac{h^{3}}{3 !} g^{\prime \prime \prime}(x)+\frac{h^{4}}{4 !} g^{(4)}(x)-\frac{h^{5}}{5 !} g^{(5)}\left(\xi_{2}\right), \\
& g(x+2 h)=g(x)+2 h g^{\prime}(x)+2 h^{2} g^{\prime \prime}(x)+\frac{8 h^{3}}{3 !} g^{\prime \prime \prime}(x)+\frac{16 h^{4}}{4 !} g^{(4)}(x)+\frac{32 h^{5}}{5 !} g^{(5)}\left(\xi_{3}\right), \\
& g(x-2 h)=g(x)-2 h g^{\prime}(x)+2 h^{2} g^{\prime \prime}(x)-\frac{8 h^{3}}{3 !} g^{\prime \prime \prime}(x)+\frac{16 h^{4}}{4 !} g^{(4)}(x)-\frac{32 h^{5}}{5 !} g^{(5)}\left(\xi_{4}\right),
\end{aligned}
$$

where $\xi_{1} \in[x, x+h], \xi_{2} \in[x-h, x], \xi_{3} \in[x, x+2 h]$ and $\xi_{4} \in[x-2 h, x]$. Then by (2.7) and (2.9), we get

$$
D_{h}^{0} g(x)-g^{\prime}(x)=\frac{h^{4}}{180}\left(g^{(5)}\left(\xi_{1}\right)+g^{(5)}\left(\xi_{2}\right)-4 g^{(5)}\left(\xi_{3}\right)-4 g^{(5)}\left(\xi_{4}\right)\right)=\mathcal{O}\left(h^{4}\right) .
$$

The equations in (2.8) imply that $D_{h}^{0} g(x)$ and $D_{h}^{2} g(x)$ approximate, respectively, the firstorder derivative $g^{\prime}(x)$ and the second-order derivative $g^{\prime \prime}(x)$ with error $\mathcal{O}\left(h^{4}\right)$.

\section{Explicit one-step schemes for FBSDEs}

We introduce the following regular time partition over interval $[0, T]$

$$
0=t_{0}<t_{1}<\cdots<t_{N}=T .
$$

Then for $n=0,1, \cdots, N-1$, we define

$$
\Delta t_{n}=t_{n+1}-t_{n}, \quad \Delta W_{n}=W_{t_{n+1}}-W_{t_{n}} .
$$




\subsection{Numerical schemes for SDEs}

In this subsection, we introduce some Itô-Taylor type schemes for solving SDEs [16].

Let $X^{n}$ denote the approximation of the solution $X_{t}$ of SDE in (1.1) at time $t=t_{n}$, and define $b^{n}=b\left(t_{n}, X^{n}\right)$ and $\sigma^{n}=\sigma\left(t_{n}, X^{n}\right)$. Then we present the following five Itô-Taylor schemes for SDEs.

1. The Euler Scheme:

$$
X^{n+1}=X^{n}+b^{n} \Delta t_{n}+\sigma^{n} \Delta W_{n}
$$

2. The Milstein scheme:

$$
X^{n+1}=X^{n}+b^{n} \Delta t_{n}+\sigma^{n} \Delta W_{n}+\frac{1}{2} \sigma^{n} \sigma_{x}^{n}\left(\left(\Delta W_{n}\right)^{2}-\Delta t_{n}\right) .
$$

3. The weak order 2.0 Itô-Taylor scheme:

$$
\begin{aligned}
X^{n+1}=X^{n} & +b^{n} \Delta t_{n}+\sigma^{n} \Delta W_{n}+\frac{1}{2} \sigma^{n} \sigma_{x}^{n}\left(\left(\Delta W_{n}\right)^{2}-\Delta t_{n}\right) \\
& +\frac{1}{2}\left(b_{t}^{n}+b^{n} b_{x}^{n}+\frac{1}{2}\left(\sigma^{n}\right)^{2} b_{x x}^{n}\right)\left(\Delta t_{n}\right)^{2} \\
& +\frac{1}{2}\left(\sigma^{n} b_{x}^{n}+\sigma_{t}^{n}+b^{n} \sigma_{x}^{n}+\frac{1}{2}\left(\sigma^{n}\right)^{2} \sigma_{x x}^{n}\right) \Delta t_{n} \Delta W_{n} .
\end{aligned}
$$

4. The weak order 3.0 Itô-Taylor scheme:

$$
\begin{aligned}
X^{n+1}=X^{n} & +b^{n} \Delta t_{n}+\sigma^{n} \Delta W_{n}+\frac{1}{2} L^{1} \sigma^{n}\left(\left(\Delta W_{n}\right)^{2}-\Delta t_{n}\right) \\
& +L^{1} b^{n} \xi_{n}+\frac{1}{2} L^{0} b^{n}\left(\Delta t_{n}\right)^{2}+L^{0} \sigma^{n}\left(\Delta W_{n} \Delta t_{n}-\xi_{n}\right) \\
& +\frac{1}{6}\left(L^{0} L^{0} \sigma^{n}+L^{0} L^{1} b^{n}+L^{1} L^{0} b^{n}\right) \Delta W_{n}\left(\Delta t_{n}\right)^{2} \\
& +\frac{1}{6}\left(L^{1} L^{1} b^{n}+L^{1} L^{0} \sigma^{n}+L^{0} L^{1} \sigma^{n}\right)\left(\left(\Delta W_{n}\right)^{2}-\Delta t_{n}\right) \Delta t_{n} \\
& +\frac{1}{6} L^{0} L^{0} b^{n}\left(\Delta t_{n}\right)^{3}+\frac{1}{6} L^{1} L^{1} \sigma^{n}\left(\left(\Delta W_{n}\right)^{2}-3 \Delta t_{n}\right) \Delta W_{n} .
\end{aligned}
$$

5. The weak order 4.0 Itô-Taylor scheme:

$$
\begin{aligned}
X^{n+1}=X^{n} & +b^{n} \Delta t_{n}+\sigma^{n} \Delta W_{n}+\frac{1}{2} L^{0} b^{n}\left(\Delta t_{n}\right)^{2}+L^{1} b^{n} \xi_{n}+L^{0} \sigma^{n}\left(\Delta W_{n} \Delta t_{n}-\xi_{n}\right) \\
& +L^{1} L^{1} b^{n}\left(2 \Delta W_{n} \xi_{n}-\frac{5}{6}\left(\Delta W_{n}\right)^{2} \Delta t_{n}-\frac{1}{6}\left(\Delta t_{n}\right)^{2}\right) \\
& +\frac{1}{6}\left(L^{0} L^{0} \sigma^{n}+L^{0} L^{1} b^{n}+L^{1} L^{0} b^{n}\right) \Delta W_{n}\left(\Delta t_{n}\right)^{2} \\
& +\frac{1}{6} L^{0} L^{0} b^{n}\left(\Delta t_{n}\right)^{3}+\frac{1}{24} L^{0} L^{0} L^{0} b^{n}\left(\Delta t_{n}\right)^{4} \\
& +\frac{1}{24} L^{1} L^{1} L^{1} b^{n} \Delta W_{n}\left(\left(\Delta W_{n}\right)^{2}-3 \Delta t_{n}\right) \Delta t_{n}
\end{aligned}
$$




$$
\begin{aligned}
& +\frac{1}{24}\left(L^{1} L^{0} L^{0} b^{n}+L^{0} L^{1} L^{0} b^{n}+L^{0} L^{0} L^{1} b^{n}+L^{0} L^{0} L^{0} \sigma^{n}\right) \Delta W_{n}\left(\Delta t_{n}\right)^{3} \\
& +\frac{1}{24}\left(L^{1} L^{1} L^{0} b^{n}+L^{0} L^{1} L^{1} b^{n}+L^{1} L^{0} L^{1} b^{n}\right)\left(\left(\Delta W_{n}\right)^{2}-\Delta t_{n}\right)\left(\Delta t_{n}\right)^{2} .
\end{aligned}
$$

Here $\xi_{n}$ and $\Delta W_{n}$ are correlated normal random variables satisfying

$$
\xi_{n} \sim N\left(0, \frac{1}{3}\left(\Delta t_{n}\right)^{3}\right), \quad \mathbb{E}\left[\Delta W_{n} \xi_{n}\right]=\frac{1}{2}\left(\Delta t_{n}\right)^{2} .
$$

\subsection{Discretisation of FBSDEs}

Let $\left(X_{t}, Y_{t}, Z_{t}\right)$ be the unique solution of (1.1), then for $n=0,1, \cdots, N-1$, we get

$$
Y_{t_{n}}=Y_{t_{n+1}}+\int_{t_{n}}^{t_{n+1}} f\left(s, X_{s}, Y_{s}, Z_{s}\right) d s-\int_{t_{n}}^{t_{n+1}} Z_{s} d W_{s}
$$

Taking the conditional expectation $\mathbb{E}_{t_{n}}^{x}[\cdot]=\mathbb{E}\left[\cdot \mid \mathcal{F}_{t_{n}}, X_{t_{n}}=x\right]$ on both sides of (3.6), we obtain

$$
Y_{t_{n}}=\mathbb{E}_{t_{n}}^{x}\left[Y_{t_{n+1}}\right]+\int_{t_{n}}^{t_{n+1}} \mathbb{E}_{t_{n}}^{x}\left[f\left(s, X_{s}, Y_{s}, Z_{s}\right)\right] d s
$$

By the Feynman-Kac formula (2.2), we have

$$
Y_{t}=u\left(t, X_{t}\right), \quad Z_{t}=\left(u_{x}^{\prime} \sigma\right)\left(t, X_{t}\right),
$$

where $u$ is the smooth solution of (2.3). Then by (2.3), (2.4) and (3.8), we deduce

$$
L^{0} u\left(t, X_{t}\right)=-f\left(t, X_{t}, u\left(t, X_{t}\right),\left(u_{x}^{\prime} \sigma\right)\left(t, X_{t}\right)\right)=-f\left(t, X_{t}, Y_{t}, Z_{t}\right),
$$

i.e., $u^{(0)}\left(t, X_{t}\right)=-f\left(t, X_{t}, Y_{t}, Z_{t}\right)$. Assume that $u \in \mathfrak{g}_{b}^{(0)_{5}}$, and then by Theorem 2.1 and the fact $\mathbb{E}_{t_{n}}^{x}[\cdot]=\mathbb{E}_{t_{n}}^{x}\left[\mathbb{E}\left[\cdot \mid \mathcal{F}_{t_{n}}\right]\right]$, we get

$$
\begin{aligned}
& \mathbb{E}_{t_{n}}^{x}\left[f\left(s, X_{s}, Y_{s}, Z_{s}\right)\right]=-\mathbb{E}_{t_{n}}^{x}\left[u^{(0)}\left(s, X_{s}\right)\right]=-\mathbb{E}_{t_{n}}^{x}\left[\mathbb{E}\left[u^{(0)}\left(s, X_{s}\right) \mid \mathcal{F}_{t_{n}}\right]\right] \\
= & -\left(u_{t_{n}}^{(0)}+\left(s-t_{n}\right) u_{t_{n}}^{(0)_{2}}+\frac{\left(s-t_{n}\right)^{2}}{2 !} u_{t_{n}}^{(0)_{3}}+\frac{\left(s-t_{n}\right)^{3}}{3 !} u_{t_{n}}^{(0)_{4}}+\mathcal{O}\left(\left(s-t_{n}\right)^{4}\right)\right),
\end{aligned}
$$

which leads to

$$
\begin{aligned}
& \int_{t_{n}}^{t_{n+1}} \mathbb{E}_{t_{n}}^{x}\left[f\left(s, X_{s}, Y_{s}, Z_{s}\right)\right] d s \\
= & -\left(\Delta t_{n} u_{t_{n}}^{(0)}+\frac{\left(\Delta t_{n}\right)^{2}}{2 !} u_{t_{n}}^{(0)_{2}}+\frac{\left(\Delta t_{n}\right)^{3}}{3 !} u_{t_{n}}^{(0)_{3}}+\frac{\left(\Delta t_{n}\right)^{4}}{4 !} u_{t_{n}}^{(0)_{4}}+\mathcal{O}\left(\left(\Delta t_{n}\right)^{5}\right)\right) .
\end{aligned}
$$

Let $A$ denote the approximation of $\int_{t_{n}}^{t_{n+1}} \mathbb{E}_{t_{n}}^{x}\left[f\left(s, X_{s}, Y_{s}, Z_{s}\right)\right] d s$, and we write

$$
\int_{t_{n}}^{t_{n+1}} \mathbb{E}_{t_{n}}^{x}\left[f\left(s, X_{s}, Y_{s}, Z_{s}\right)\right] d s=A+R_{Y 1}^{n},
$$


where $R_{Y 1}^{n}$ is the approximation error and

$$
A=-\left(\theta \Delta t_{n} u_{t_{n}}^{(0)}+(1-\theta) \Delta t_{n} \mathbb{E}_{t_{n}}^{x}\left[u_{t_{n+1}}^{(0)}\right]+\left(\Delta t_{n}\right)^{2} \alpha u_{t_{n}}^{(0)_{2}}+\left(\Delta t_{n}\right)^{2} \beta \mathbb{E}_{t_{n}}^{x}\left[u_{t_{n+1}}^{(0)_{2}}\right]\right)
$$

with $\theta \in[0,1], \alpha$ and $\beta$ being parameters. Note that, by Theorem 2.1, we deduce

$$
\begin{aligned}
A=- & \left(\theta \Delta t_{n} u_{t_{n}}^{(0)}+(1-\theta) \Delta t_{n}\left[u_{t_{n}}^{(0)}+\Delta t_{n} u_{t_{n}}^{(0)_{2}}+\frac{\left(\Delta t_{n}\right)^{2}}{2 !} u_{t_{n}}^{(0)_{3}}+\frac{\left(\Delta t_{n}\right)^{3}}{3 !} u_{t_{n}}^{(0)_{4}}\right]\right. \\
& \left.+\left(\Delta t_{n}\right)^{2} \alpha u_{t_{n}}^{(0)_{2}}+\left(\Delta t_{n}\right)^{2} \beta\left[u_{t_{n}}^{(0)_{2}}+\Delta t_{n} u_{t_{n}}^{(0)_{3}}+\frac{\left(\Delta t_{n}\right)^{2}}{2 !} u_{t_{n}}^{(0)_{4}}\right]+\mathcal{O}\left(\left(\Delta t_{n}\right)^{5}\right)\right) \\
=- & \left(\Delta t_{n} u_{t_{n}}^{(0)}+(1-\theta+\alpha+\beta)\left(\Delta t_{n}\right)^{2} u_{t_{n}}^{(0)_{2}}+\left(\frac{1-\theta}{2}+\beta\right)\left(\Delta t_{n}\right)^{3} u_{t_{n}}^{(0)_{3}}\right. \\
& \left.+\left(\frac{1-\theta}{6}+\frac{\beta}{2}\right)\left(\Delta t_{n}\right)^{4} u_{t_{n}}^{(0)_{4}}+\mathcal{O}\left(\left(\Delta t_{n}\right)^{5}\right)\right) .
\end{aligned}
$$

Define the parameters $\alpha$ and $\beta$ in $A$ as

$$
\alpha=\frac{3 \theta-1}{6}, \quad \beta=\frac{3 \theta-2}{6},
$$

and we obtain

$$
A=-\left(\Delta t_{n} u_{t_{n}}^{(0)}+\frac{1}{2 !}\left(\Delta t_{n}\right)^{2} u_{t_{n}}^{(0)_{2}}+\frac{1}{3 !}\left(\Delta t_{n}\right)^{3} u_{t_{n}}^{(0)_{3}}+\frac{\theta}{12}\left(\Delta t_{n}\right)^{4} u_{t_{n}}^{(0)_{4}}+\mathcal{O}\left(\left(\Delta t_{n}\right)^{5}\right)\right) .
$$

Then by (3.10), (3.11) and (3.15), we deduce

$$
\begin{aligned}
R_{Y 1}^{n} & =\int_{t_{n}}^{t_{n+1}} \mathbb{E}_{t_{n}}^{x}\left[f\left(s, X_{s}, Y_{s}, Z_{s}\right)\right] d s-A \\
& =\left(\frac{\theta}{12}-\frac{1}{24}\right)\left(\Delta t_{n}\right)^{4} u_{t_{n}}^{(0)_{4}}+\mathcal{O}\left(\left(\Delta t_{n}\right)^{5}\right),
\end{aligned}
$$

which implies that

$$
R_{Y 1}^{n}= \begin{cases}\mathcal{O}\left((\Delta t)^{4}\right), & \theta \neq 0.5 \\ \mathcal{O}\left((\Delta t)^{5}\right), & \theta=0.5 .\end{cases}
$$

By using (3.7), (3.11), (3.12) and (3.14), we arrive at

$$
\begin{aligned}
Y_{t_{n}}=\mathbb{E}_{t_{n}}^{x} & {\left[Y_{t_{n+1}}\right]-\Delta t_{n}\left(\theta u_{t_{n}}^{(0)}+(1-\theta) \mathbb{E}_{t_{n}}^{x}\left[u_{t_{n+1}}^{(0)}\right]\right) } \\
& -\left(\Delta t_{n}\right)^{2}\left(\frac{3 \theta-1}{6} u_{t_{n}}^{(0)_{2}}+\frac{3 \theta-2}{6} \mathbb{E}_{t_{n}}^{x}\left[u_{t_{n+1}}^{(0)_{2}}\right]\right)+R_{Y 1}^{n} .
\end{aligned}
$$


To write $u_{t_{n}}^{(0)_{2}}$ in (3.17) in an explicit form, we apply the operator $L^{0}$ to (3.9) to get

$$
\begin{aligned}
-u_{t}^{(0)_{2}}= & L^{0} f\left(t, X_{t}, u_{t}, \sigma_{t} \frac{\partial u_{t}}{\partial x}\right)=\left(\frac{\partial}{\partial t}+b_{t} \frac{\partial}{\partial x}+\frac{1}{2} \sigma_{t}^{2} \frac{\partial^{2}}{\partial x^{2}}\right) f\left(t, X_{t}, u_{t}, \sigma_{t} \frac{\partial u_{t}}{\partial x}\right) \\
=f_{t, 1}^{\prime} & +f_{t, 3}^{\prime} \frac{\partial u_{t}}{\partial t}+f_{t, 4}^{\prime}\left(\frac{\partial \sigma_{t}}{\partial t} \frac{\partial u_{t}}{\partial x}+\sigma_{t} \frac{\partial^{2} u_{t}}{\partial x \partial t}\right) \\
& +b_{t}\left(f_{t, 2}^{\prime}+f_{t, 3}^{\prime} \frac{\partial u_{t}}{\partial x}+f_{t, 4}^{\prime}\left(\frac{\partial \sigma_{t}}{\partial x} \frac{\partial u_{t}}{\partial x}+\sigma_{t} \frac{\partial^{2} u_{t}}{\partial x^{2}}\right)\right) \\
& +\frac{1}{2} \sigma_{t}^{2} \frac{\partial}{\partial x}\left(f_{t, 2}^{\prime}+f_{t, 3}^{\prime} \frac{\partial u_{t}}{\partial x}+f_{t, 4}^{\prime}\left(\frac{\partial \sigma_{t}}{\partial x} \frac{\partial u_{t}}{\partial x}+\sigma_{t} \frac{\partial^{2} u_{t}}{\partial x^{2}}\right)\right),
\end{aligned}
$$

where $b_{t}=b\left(t, X_{t}\right)$ and $\sigma_{t}=\sigma\left(t, X_{t}\right)$. Here and in the sequel, we define

$$
\begin{array}{ll}
f_{t}=f\left(t, X_{t}, u_{t}, \sigma_{t} \frac{\partial u_{t}}{\partial x}\right), & f_{t, 1}^{\prime}=\frac{\partial f}{\partial t}\left(t, X_{t}, u_{t}, \sigma_{t} \frac{\partial u_{t}}{\partial x}\right), \\
f_{t, 2}^{\prime}=\frac{\partial f}{\partial x}\left(t, X_{t}, u_{t}, \sigma_{t} \frac{\partial u_{t}}{\partial x}\right), & f_{t, 3}^{\prime}=\frac{\partial f}{\partial y}\left(t, X_{t}, u_{t}, \sigma_{t} \frac{\partial u_{t}}{\partial x}\right), \\
f_{t, 4}^{\prime}=\frac{\partial f}{\partial z}\left(t, X_{t}, u_{t}, \sigma_{t} \frac{\partial u_{t}}{\partial x}\right), & f_{t, 22}^{\prime \prime}=\frac{\partial^{2} f}{\partial x^{2}}\left(t, X_{t}, u_{t}, \sigma_{t} \frac{\partial u_{t}}{\partial x}\right),
\end{array}
$$

and define $b_{t, i}^{\prime}, \sigma_{t, i}^{\prime}, b_{t, i j}^{\prime \prime}, \sigma_{t, i j}^{\prime \prime},(i, j=1,2)$, and $f_{t, i j}^{\prime \prime},(i, j=2,3,4)$ in a similar way. By (3.9) and the definition of $L^{0}$, it holds that

$$
\frac{\partial u_{t}}{\partial t}=-\left(b_{t} \frac{\partial u_{t}}{\partial x}+\frac{1}{2} \sigma_{t}^{2} \frac{\partial^{2} u_{t}}{\partial x^{2}}+f\left(t, X_{t}, u_{t}, \sigma_{t} \frac{\partial u_{t}}{\partial x}\right)\right)
$$

which leads to

$$
\begin{aligned}
\frac{\partial^{2} u_{t}}{\partial x \partial t}=- & \frac{\partial}{\partial x}\left(b_{t} \frac{\partial u_{t}}{\partial x}+\frac{1}{2} \sigma_{t}^{2} \frac{\partial^{2} u_{t}}{\partial x^{2}}+f\left(t, X_{t}, u_{t}, \sigma_{t} \frac{\partial u_{t}}{\partial x}\right)\right) \\
=- & \left(\frac{\partial b_{t}}{\partial x} \frac{\partial u_{t}}{\partial x}+b_{t} \frac{\partial^{2} u_{t}}{\partial x^{2}}+\sigma_{t} \frac{\partial \sigma_{t}}{\partial x} \frac{\partial^{2} u_{t}}{\partial x^{2}}+\frac{1}{2} \sigma_{t}^{2} \frac{\partial^{3} u_{t}}{\partial x^{3}}\right. \\
& \left.+f_{t, 2}^{\prime}+f_{t, 3}^{\prime} \frac{\partial u_{t}}{\partial x}+f_{t, 4}^{\prime}\left(\frac{\partial \sigma_{t}}{\partial x} \frac{\partial u_{t}}{\partial x}+\sigma_{t} \frac{\partial^{2} u_{t}}{\partial x^{2}}\right)\right) .
\end{aligned}
$$

Inserting (3.19) and (3.20) into (3.18), we deduce

$$
\begin{aligned}
-u_{t}^{(0)_{2}=f_{t, 1}^{\prime}} & +f_{t, 2}^{\prime}\left(b_{t}-\sigma_{t} f_{t, 4}^{\prime}\right)-f_{t, 3}^{\prime} f_{t}+f_{t, 4}^{\prime}\left(\sigma_{t, 1}^{\prime}+b_{t} \sigma_{t, 2}^{\prime}+\frac{1}{2} \sigma_{t}^{2} \sigma_{t, 22}^{\prime \prime}\right) \frac{\partial u_{t}}{\partial x} \\
& -f_{t, 4}^{\prime} \sigma_{t}\left(\left(b_{t, 2}^{\prime}+f_{t, 3}^{\prime}+f_{t, 4}^{\prime} \sigma_{t, 2}^{\prime}\right) \frac{\partial u_{t}}{\partial x}+\sigma_{t} f_{t, 4}^{\prime} \frac{\partial^{2} u_{t}}{\partial x^{2}}\right) \\
& +\frac{1}{2} \sigma_{t}^{2}\left(f_{t, 22}^{\prime \prime}+2 f_{t, 23}^{\prime \prime} \frac{\partial u_{t}}{\partial x}+2 f_{t, 24}^{\prime \prime}\left(\sigma_{t, 2}^{\prime} \frac{\partial u_{t}}{\partial x}+\sigma_{t} \frac{\partial^{2} u_{t}}{\partial x^{2}}\right)+f_{t, 33}^{\prime \prime}\left(\frac{\partial u_{t}}{\partial x}\right)^{2}\right.
\end{aligned}
$$




$$
\begin{aligned}
& \left.\quad+2 f_{t, 34}^{\prime \prime} \frac{\partial u_{t}}{\partial x}\left(\sigma_{t, 2}^{\prime} \frac{\partial u_{t}}{\partial x}+\sigma_{t} \frac{\partial^{2} u_{t}}{\partial x^{2}}\right)+f_{t, 44}^{\prime \prime}\left(\sigma_{t, 2}^{\prime} \frac{\partial u_{t}}{\partial x}+\sigma_{t} \frac{\partial^{2} u_{t}}{\partial x^{2}}\right)^{2}\right) \\
& =F\left(t, X_{t}, u_{t}, \frac{\partial u_{t}}{\partial x}, \frac{\partial^{2} u_{t}}{\partial x^{2}}\right),
\end{aligned}
$$

where $F(t, x, y, z, \gamma):[0, T] \times \mathbb{R} \times \mathbb{R} \times \mathbb{R} \times \mathbb{R} \rightarrow \mathbb{R}$. Let

$$
F_{t}=F\left(t, X_{t}, u_{t}, \frac{\partial u_{t}}{\partial x}, \frac{\partial^{2} u_{t}}{\partial x^{2}}\right)
$$

then by (3.9), (3.17) and (3.21), we have

$$
\begin{aligned}
Y_{t_{n}}=\mathbb{E}_{t_{n}}^{x} & {\left[Y_{t_{n+1}}\right]+\theta \Delta t_{n} f_{t_{n}}+(1-\theta) \Delta t_{n} \mathbb{E}_{t_{n}}^{x}\left[f_{t_{n+1}}\right] } \\
& +\frac{(3 \theta-1)\left(\Delta t_{n}\right)^{2}}{6} F_{t_{n}}+\frac{(3 \theta-2)\left(\Delta t_{n}\right)^{2}}{6} \mathbb{E}_{t_{n}}^{x}\left[F_{t_{n+1}}\right]+R_{Y 1}^{n} .
\end{aligned}
$$

By (2.7) and (3.8), the solution $Z_{t}$ can be represented as

$$
Z_{t}=\sigma_{t} \frac{\partial u_{t}}{\partial x}=\sigma_{t} D_{h}^{0} u_{t}+\mathcal{O}\left(h^{4}\right)
$$

where $D_{h}^{0} u_{t}=D_{h}^{0} Y_{t}\left(X_{t}\right)$. Then we define

$$
\begin{array}{ll}
f_{t, h}=f\left(t, X_{t}, u_{t}, \sigma_{t} D_{h}^{0} u_{t}\right), & \\
f_{t, h, i}^{\prime}=f_{t, i}^{\prime}\left(t, X_{t}, u_{t}, \sigma_{t} D_{h}^{0} u_{t}\right), & (i=1,2,3,4), \\
f_{t, h, i j}^{\prime \prime}=f_{t, i j}^{\prime \prime}\left(t, X_{t}, u_{t}, \sigma_{t} D_{h}^{0} u_{t}\right), & (i, j=2,3,4),
\end{array}
$$

and denote by

$$
\begin{aligned}
F_{t, h}= & f_{t, h, 1}^{\prime}+f_{t, h, 2}^{\prime}\left(b_{t}-\sigma_{t} f_{t, h, 4}^{\prime}\right)-f_{t, h, 3}^{\prime} f_{t, h}+f_{t, h, 4}^{\prime}\left(\sigma_{t, 1}^{\prime}+b_{t} \sigma_{t, 2}^{\prime}+\frac{1}{2} \sigma_{t}^{2} \sigma_{t, 22}^{\prime \prime}\right) D_{h}^{0} u_{t} \\
& -f_{t, h, 4}^{\prime} \sigma_{t}\left(\left(b_{t, 2}^{\prime}+f_{t, h, 3}^{\prime}+f_{t, h, 4}^{\prime} \sigma_{t, 2}^{\prime}\right) D_{h}^{0} u_{t}+\sigma_{t} f_{t, h, 4}^{\prime} D_{h}^{2} u_{t}\right) \\
& +\frac{1}{2} \sigma_{t}^{2}\left(f_{t, h, 22}^{\prime \prime}+2 f_{t, h, 23}^{\prime \prime} D_{h}^{0} u_{t}+2 f_{t, h, 24}^{\prime \prime}\left(\sigma_{t, 2}^{\prime} D_{h}^{0} u_{t}+\sigma_{t} D_{h}^{2} u_{t}\right)+f_{t, h, 33}^{\prime \prime}\left(D_{h}^{0} u_{t}\right)^{2}\right. \\
& \left.+2 f_{t, h, 34}^{\prime \prime} D_{h}^{0} u_{t}\left(\sigma_{t, 2}^{\prime} D_{h}^{0} u_{t}+\sigma_{t} D_{h}^{2} u_{t}\right)+f_{t, h, 44}^{\prime \prime}\left(\sigma_{t, 2}^{\prime} D_{h}^{0} u_{t}+\sigma_{t} D_{h}^{2} u_{t}\right)^{2}\right),
\end{aligned}
$$

where $D_{h}^{2} u_{t}=D_{h}^{2} Y_{t}\left(X_{t}\right)$. Then by (3.22), (3.24) and (3.25), we get

$$
\begin{aligned}
Y_{t_{n}}=\mathbb{E}_{t_{n}}^{x}\left[Y_{t_{n+1}}\right]+\theta \Delta t_{n} f_{t_{n}, h}+(1-\theta) \Delta t_{n} \mathbb{E}_{t_{n}}^{x}\left[f_{t_{n+1}, h}\right] \\
+\frac{(3 \theta-1)\left(\Delta t_{n}\right)^{2}}{6} F_{t_{n}, h}+\frac{(3 \theta-2)\left(\Delta t_{n}\right)^{2}}{6} \mathbb{E}_{t_{n}}^{x}\left[F_{t_{n+1}, h}\right]+R_{Y 1}^{n}+R_{Y 2}^{n},
\end{aligned}
$$


where

$$
\begin{aligned}
R_{Y 2}^{n}=\theta & \Delta t_{n}\left(f_{t_{n}}-f_{t_{n}, h}\right)+(1-\theta) \Delta t_{n} \mathbb{E}_{t_{n}}^{x}\left[f_{t_{n+1}}-f_{t_{n+1}, h}\right] \\
& +\frac{(3 \theta-1)\left(\Delta t_{n}\right)^{2}}{6}\left(F_{t_{n}}-F_{t_{n}, h}\right)+\frac{(3 \theta-2)\left(\Delta t_{n}\right)^{2}}{6} \mathbb{E}_{t_{n}}^{x}\left[F_{t_{n+1}}-F_{t_{n+1}, h}\right] .
\end{aligned}
$$

To solve $Y_{t_{n}}$ in an explicit way, we first use Euler scheme to approximate $Y_{t_{n}}$ in $f_{t_{n}, h}$ and $F_{t_{n}, h}$, which yields

$$
Y_{t_{n}}=\mathbb{E}_{t_{n}}^{x}\left[Y_{t_{n+1}}\right]+\Delta t_{n} \mathbb{E}_{t_{n}}^{x}\left[f_{t_{n+1}, h}\right]+R_{y 1}^{n}=\bar{Y}_{t_{n}}+R_{y 1}^{n},
$$

where $R_{y 1}^{n}$ is the approximation error and

$$
\bar{Y}_{t_{n}}=\mathbb{E}_{t_{n}}^{x}\left[Y_{t_{n+1}}\right]+\Delta t_{n} \mathbb{E}_{t_{n}}^{x}\left[f_{t_{n+1}, h}\right]
$$

Moreover, we let

$$
\bar{f}_{t_{n}, h}=f\left(t_{n}, x, \bar{Y}_{t_{n}}(x), D_{h}^{0} \bar{Y}_{t_{n}}(x) \sigma\left(t_{n}, x\right)\right),
$$

and further approximate $Y_{t_{n}}$ in $f_{t_{n}, h}$ and $F_{t_{n}, h}$ by Crank-Nicolson scheme

$$
Y_{t_{n}}=\mathbb{E}_{t_{n}}^{x}\left[Y_{t_{n+1}}\right]+\frac{1}{2} \Delta t_{n} \bar{f}_{t_{n}, h}+\frac{1}{2} \Delta t_{n} \mathbb{E}_{t_{n}}^{x}\left[f_{t_{n+1}, h}\right]+R_{y 2}^{n}=\overline{\bar{Y}}_{t_{n}}+R_{y 2}^{n}
$$

where $R_{y 2}^{n}$ is the approximation error and

$$
\overline{\bar{Y}}_{t_{n}}=\mathbb{E}_{t_{n}}^{x}\left[Y_{t_{n+1}}\right]+\frac{1}{2} \Delta t_{n} \bar{f}_{t_{n}, h}+\frac{1}{2} \Delta t_{n} \mathbb{E}_{t_{n}}^{x}\left[f_{t_{n+1}, h}\right]
$$

Denote by

$$
\begin{aligned}
& \overline{\bar{f}}_{t_{n}, h}=f\left(t_{n}, x, \overline{\bar{Y}}_{t_{n}}(x), D_{h}^{0} \overline{\bar{Y}}_{t_{n}}(x) \sigma\left(t_{n}, x\right)\right), \\
& \overline{\bar{F}}_{t_{n}, h}=F_{t_{n}, h}\left(t_{n}, x, \overline{\bar{Y}}_{t_{n}}(x), D_{h}^{0} \overline{\bar{Y}}_{t_{n}}(x), D_{h}^{2} \overline{\bar{Y}}_{t_{n}}(x)\right) .
\end{aligned}
$$

Then by (3.26) and (3.33), we get the first reference equation for solving $Y_{t}$

$$
\begin{aligned}
Y_{t_{n}}=\mathbb{E}_{t_{n}}^{x} & {\left[Y_{t_{n+1}}\right]+\theta \Delta t_{n} \overline{\bar{f}}_{t_{n}, h}+(1-\theta) \Delta t_{n} \mathbb{E}_{t_{n}}^{x}\left[f_{t_{n+1}, h}\right] } \\
& +\frac{(3 \theta-1)\left(\Delta t_{n}\right)^{2}}{6} \overline{\bar{F}}_{t_{n}, h}+\frac{(3 \theta-2)\left(\Delta t_{n}\right)^{2}}{6} \mathbb{E}_{t_{n}}^{x}\left[F_{t_{n+1}, h}\right]+R_{y 3}^{n},
\end{aligned}
$$

where $R_{y 3}^{n}=R_{Y 1}^{n}+R_{Y 2}^{n}+R_{Y 3}^{n}$ with

$$
R_{Y 3}^{n}=\theta \Delta t_{n}\left(f_{t_{n}, h}-\overline{\bar{f}}_{t_{n}, h}\right)+\frac{(3 \theta-1)\left(\Delta t_{n}\right)^{2}}{6}\left(F_{t_{n}, h}-\overline{\bar{F}}_{t_{n}, h}\right) .
$$


Now based on (3.34), we turn to constructing the second reference equation for $Y_{t}$. To this end, we take $\theta=\frac{1}{2}$ in (3.26) to obtain

$$
\begin{aligned}
Y_{t_{n}}=\mathbb{E}_{t_{n}}^{x} & {\left[Y_{t_{n+1}}\right]+\frac{1}{2} \Delta t_{n} f_{t_{n}, h}+\frac{1}{2} \Delta t_{n} \mathbb{E}_{t_{n}}^{x}\left[f_{t_{n+1}, h}\right] } \\
& +\frac{1}{12}\left(\Delta t_{n}\right)^{2} F_{t_{n}, h}-\frac{1}{12}\left(\Delta t_{n}\right)^{2} \mathbb{E}_{t_{n}}^{x}\left[F_{t_{n+1}, h}\right]+\hat{R}_{Y 1}^{n}+\hat{R}_{Y 2}^{n},
\end{aligned}
$$

where $\hat{R}_{Y 1}^{n}$ and $\hat{R}_{Y 2}^{n}$ are the truncation error terms $R_{Y 1}^{n}$ and $R_{Y 2}^{n}$ with $\theta=\frac{1}{2}$. By removing the truncation error term $R_{y 3}^{n}$ in (3.34), we have

$$
\begin{aligned}
\hat{Y}_{t_{n}}=\mathbb{E}_{t_{n}}^{x}\left[Y_{t_{n+1}}\right]+\theta \Delta t_{n} \overline{\bar{f}}_{t_{n}, h}+(1-\theta) \Delta t_{n} \mathbb{E}_{t_{n}}^{x}\left[f_{t_{n+1}, h}\right] \\
+\frac{(3 \theta-1)\left(\Delta t_{n}\right)^{2}}{6} \overline{\bar{F}}_{t_{n}, h}+\frac{(3 \theta-2)\left(\Delta t_{n}\right)^{2}}{6} \mathbb{E}_{t_{n}}^{x}\left[F_{t_{n+1}, h}\right] .
\end{aligned}
$$

Let

$$
\begin{aligned}
& \hat{f}_{t_{n}, h}=f\left(t_{n}, x, \hat{Y}_{t_{n}}(x), D_{h}^{0} \hat{Y}_{t_{n}}(x) \sigma\left(t_{n}, x\right)\right), \\
& \hat{F}_{t_{n}, h}=F_{t_{n}, h}\left(t_{n}, x, \hat{Y}_{t_{n}}(x), D_{h}^{0} \hat{Y}_{t_{n}}(x), D_{h}^{2} \hat{Y}_{t_{n}}(x)\right) .
\end{aligned}
$$

Then by (3.36) and (3.38), we get the second reference equation for solving $Y_{t}$

$$
\begin{aligned}
Y_{t_{n}}=\mathbb{E}_{t_{n}}^{x} & {\left[Y_{t_{n+1}}\right]+\frac{1}{2} \Delta t_{n} \hat{f}_{t_{n}, h}+\frac{1}{2} \Delta t_{n} \mathbb{E}_{t_{n}}^{x}\left[f_{t_{n+1}, h}\right] } \\
& +\frac{1}{12}\left(\Delta t_{n}\right)^{2} \hat{F}_{t_{n}, h}-\frac{1}{12}\left(\Delta t_{n}\right)^{2} \mathbb{E}_{t_{n}}^{x}\left[F_{t_{n+1}, h}\right]+R_{y 4}^{n},
\end{aligned}
$$

where $R_{y 4}^{n}=\hat{R}_{Y 1}^{n}+\hat{R}_{Y 2}^{n}+\hat{R}_{Y 3}^{n}$ with

$$
\hat{R}_{Y 3}^{n}=\frac{1}{2} \Delta t_{n}\left(f_{t_{n}, h}-\hat{f}_{t_{n}, h}\right)+\frac{1}{12}\left(\Delta t_{n}\right)^{2}\left(F_{t_{n}, h}-\hat{F}_{t_{n}, h}\right) .
$$

\subsection{The explicit one-step schemes for FBSDEs}

Let $\left(X^{n}, Y^{n}, Z^{n}\right)$ denote the numerical approximation of the solution $\left(X_{t}, Y_{t}, Z_{t}\right)$ of the FBSDE (1.1) at time level $t=t_{n},(n=N, \cdots, 0)$. For simplicity, we define $f^{n, h}$ and $F^{n, h}$ by

$$
\begin{aligned}
& f^{n, h}=f\left(t_{n}, X^{n}, Y^{n}\left(X^{n}\right), D_{h}^{0} Y^{n}\left(X^{n}\right) \sigma\left(t_{n}, X^{n}\right)\right), \\
& F^{n, h}=F\left(t_{n}, X^{n}, Y^{n}\left(X^{n}\right), D_{h}^{0} Y^{n}\left(X^{n}\right), D_{h}^{2} Y^{n}\left(X^{n}\right)\right) .
\end{aligned}
$$

Based on the reference equations (3.23), (3.29), (3.32) and (3.34), we first propose the following explicit third order one-step scheme for solving the FBSDE (1.1). 
Scheme 3.1. Given random variable $Y^{N}$, for $n=N-1, \cdots, 0$, we solve the random variables $Y^{n}=Y^{n}\left(X^{n}\right)$ and $Z^{n}=Z^{n}\left(X^{n}\right)$ by

$$
\begin{aligned}
\bar{Y}^{n}= & \mathbb{E}_{t_{n}}^{x}\left[Y^{n+1}\right]+\Delta t_{n} \mathbb{E}_{t_{n}}^{x}\left[f^{n+1, h}\right], \\
\overline{Y^{n}}= & \mathbb{E}_{t_{n}}^{x}\left[Y^{n+1}\right]+\frac{1}{2} \Delta t_{n} \bar{f}^{n, h}+\frac{1}{2} \Delta t_{n} \mathbb{E}_{t_{n}}^{x}\left[f^{n+1, h}\right], \\
Y^{n}= & \mathbb{E}_{t_{n}}^{x}\left[Y^{n+1}\right]+\theta \Delta t_{n} \overline{\bar{f}}^{n, h}+(1-\theta) \Delta t_{n} \mathbb{E}_{t_{n}}^{x}\left[f^{n+1, h}\right] \\
& \quad+\frac{(3 \theta-1)\left(\Delta t_{n}\right)^{2}}{6} \overline{\bar{F}}^{n, h}+\frac{(3 \theta-2)\left(\Delta t_{n}\right)^{2}}{6} \mathbb{E}_{t_{n}}^{x}\left[F^{n+1, h}\right], \\
Z^{n}= & D_{h}^{0} Y^{n} \sigma\left(t_{n}, X^{n}\right),
\end{aligned}
$$

where $\theta \in[0,1]$ and

$$
\begin{aligned}
& \bar{f}^{n, h}=f\left(t_{n}, X^{n}, \bar{Y}^{n}\left(X^{n}\right), D_{h}^{0} \bar{Y}^{n}\left(X^{n}\right) \sigma\left(t_{n}, X^{n}\right)\right), \\
& \overline{\bar{f}}^{n, h}=f\left(t_{n}, X^{n}, \overline{\bar{Y}}^{n}\left(X^{n}\right), D_{h}^{0} \overline{\bar{Y}}^{n}\left(X^{n}\right) \sigma\left(t_{n}, X^{n}\right)\right), \\
& \overline{\bar{F}}^{n, h}=F\left(t_{n}, X^{n}, \bar{Y}^{n}\left(X^{n}\right), D_{h}^{0} \overline{\bar{Y}}^{n}\left(X^{n}\right), D_{h}^{2} \bar{Y}^{n}\left(X^{n}\right)\right),
\end{aligned}
$$

with $X^{n}$ being solved by Itô-Taylor schemes.

Furthermore, based on the reference equations (3.23), (3.29), (3.32), (3.37) and (3.39), we construct the following explicit fourth order one-step scheme for the FBSDE (1.1).

Scheme 3.2. Given random variable $Y^{N}$, for $n=N-1, \cdots, 0$, we solve the random variables $Y^{n}=Y^{n}\left(X^{n}\right)$ and $Z^{n}=Z^{n}\left(X^{n}\right)$ by

$$
\begin{aligned}
& \bar{Y}^{n}= \mathbb{E}_{t_{n}}^{x}\left[Y^{n+1}\right]+\Delta t_{n} \mathbb{E}_{t_{n}}^{x}\left[f^{n+1, h}\right], \\
& \overline{Y^{n}}= \mathbb{E}_{t_{n}}^{x}\left[Y^{n+1}\right]+\frac{1}{2} \Delta t_{n} \bar{f}^{n, h}+\frac{1}{2} \Delta t_{n} \mathbb{E}_{t_{n}}^{x}\left[f^{n+1, h}\right], \\
& \hat{Y}^{n}= \mathbb{E}_{t_{n}}^{x}\left[Y^{n+1}\right]+\theta \Delta t_{n} \overline{\bar{f}}^{n, h}+(1-\theta) \Delta t_{n} \mathbb{E}_{t_{n}}^{x}\left[f^{n+1, h}\right] \\
& \quad+\frac{(3 \theta-1)\left(\Delta t_{n}\right)^{2}}{6} \overline{\bar{F}}^{n, h}+\frac{(3 \theta-2)\left(\Delta t_{n}\right)^{2}}{6} \mathbb{E}_{t_{n}}^{x}\left[F^{n+1, h}\right], \\
& Y^{n}= \mathbb{E}_{t_{n}}^{x}\left[Y^{n+1}\right]+\frac{1}{2} \Delta t_{n} \hat{f}^{n, h}+\frac{1}{2} \Delta t_{n} \mathbb{E}_{t_{n}}^{x}\left[f^{n+1, h}\right] \\
& \quad+\frac{1}{12}\left(\Delta t_{n}\right)^{2} \hat{F}^{n, h}-\frac{1}{12}\left(\Delta t_{n}\right)^{2} \mathbb{E}_{t_{n}}^{x}\left[F^{n+1, h}\right], \\
& Z^{n}=D_{h}^{0} Y^{n} \sigma\left(t_{n}, X^{n}\right),
\end{aligned}
$$

where $\theta \in[0,1]$ and

$$
\begin{aligned}
& \bar{f}^{n, h}=f\left(t_{n}, X^{n}, \bar{Y}^{n}\left(X^{n}\right), D_{h}^{0} \bar{Y}^{n}\left(X^{n}\right) \sigma\left(t_{n}, X^{n}\right)\right), \\
& \overline{\bar{f}}^{n, h}=f\left(t_{n}, X^{n}, \overline{\bar{Y}}_{2}^{n}\left(X^{n}\right), D_{h}^{0} \overline{\bar{Y}}_{2}^{n}\left(X^{n}\right) \sigma\left(t_{n}, X^{n}\right)\right), \\
& \overline{\bar{F}}^{n, h}=F\left(t_{n}, X^{n}, \overline{\bar{Y}}_{2}^{n}\left(X^{n}\right), D_{h}^{0} \overline{\bar{Y}}_{2}^{n}\left(X^{n}\right), D_{h}^{2} \overline{\bar{Y}}_{2}^{n}\left(X^{n}\right)\right), \\
& \hat{f}^{n, h}=f\left(t_{n}, X^{n}, \hat{Y}^{n}\left(X^{n}\right), D_{h}^{0} \hat{Y}^{n}\left(X^{n}\right) \sigma\left(t_{n}, X^{n}\right)\right), \\
& \hat{F}^{n, h}=F\left(t_{n}, X^{n}, \hat{Y}^{n}\left(X^{n}\right), D_{h}^{0} \hat{Y}^{n}\left(X^{n}\right), D_{h}^{2} \hat{Y}^{n}\left(X^{n}\right)\right),
\end{aligned}
$$


with $X^{n}$ being solved by Itô-Taylor schemes.

\subsection{Error estimates}

Now we turn to analyzing the accuracy and convergence of Schemes 3.1 and 3.2. To this end, we give the estimations of the truncation error terms $R_{y 3}^{n}$ and $R_{y 4}^{n}$ in the reference equations (3.34) and (3.39), respectively.

Assumption 3.1. The functions $b, \sigma, f$ and $\varphi$ are bounded and smooth enough with bounded derivatives.

To proceed, we first estimate the truncation error terms $R_{y 1}^{n}$ and $R_{y 2}^{n}$.

Theorem 3.1. Under Assumption 3.1, we have the following local estimates for $n=0,1, \cdots, N-1$ :

$$
\begin{aligned}
& \left|R_{y 1}^{n}\right| \leq C\left(\left(\Delta t_{n}\right)^{2}+h^{4} \Delta t_{n}\right), \\
& \left|R_{y 2}^{n}\right| \leq C\left(\left(\Delta t_{n}\right)^{3}+h^{4} \Delta t_{n}\right),
\end{aligned}
$$

where $C$ is a positive constant depending on $T$ and the upper bounds of derivatives of $b, \sigma, f$ and $\varphi$.

Proof. By (3.7) and (3.28), we have

$$
\begin{aligned}
R_{y 1}^{n} & =\int_{t_{n}}^{t_{n+1}} \mathbb{E}_{t_{n}}^{x}\left[f_{s}\right] d s-\Delta t_{n} \mathbb{E}_{t_{n}}^{x}\left[f_{t_{n+1}, h}\right] \\
& =\int_{t_{n}}^{t_{n+1}}\left(\mathbb{E}_{t_{n}}^{x}\left[f_{s}\right]-\mathbb{E}_{t_{n}}^{x}\left[f_{t_{n+1}}\right]\right) d s+\Delta t_{n}\left(\mathbb{E}_{t_{n}}^{x}\left[f_{t_{n+1}}-f_{t_{n+1}, h}\right]\right) .
\end{aligned}
$$

By Itô-Taylor expansion, it is easy to deduce (see [38])

$$
\left|\int_{t_{n}}^{t_{n+1}}\left(\mathbb{E}_{t_{n}}^{x}\left[f_{s}\right]-\mathbb{E}_{t_{n}}^{x}\left[f_{t_{n+1}}\right]\right) d s\right| \leq C\left(\Delta t_{n}\right)^{2} .
$$

Using the accuracy of $D_{h}^{0}$ in (2.7), we get

$$
\left|f_{t}-f_{t, h}\right| \leq C\left|Y_{t}\left(X_{t}\right)-D_{h}^{0} Y_{t}\left(X_{t}\right)\right| \leq C h^{4} .
$$

Eqs. (3.42)-(3.44) lead to the inequality (3.41a). By (3.7) and (3.31), we have

$$
\begin{aligned}
R_{y 2}^{n}=\int_{t_{n}}^{t_{n+1}} \mathbb{E}_{t_{n}}^{x}\left[f_{s}\right] d s-\frac{1}{2} \Delta t_{n} \bar{f}_{t_{n}, h}-\frac{1}{2} \Delta t_{n} \mathbb{E}_{t_{n}}^{x}\left[f_{t_{n+1}, h}\right] \\
=\int_{t_{n}}^{t_{n+1}}\left(\mathbb{E}_{t_{n}}^{x}\left[f_{s}\right]-\frac{1}{2} f_{t_{n}}-\frac{1}{2} \mathbb{E}_{t_{n}}^{x}\left[f_{t_{n+1}}\right]\right) d s \\
\quad+\frac{1}{2} \Delta t_{n}\left(f_{t_{n}}-f_{t_{n}, h}\right)+\frac{1}{2} \Delta t_{n}\left(f_{t_{n}, h}-\bar{f}_{t_{n}, h}\right) \\
\quad+\frac{1}{2} \Delta t_{n}\left(\mathbb{E}_{t_{n}}^{x}\left[f_{t_{n+1}}-f_{t_{n+1}, h}\right]\right) .
\end{aligned}
$$


By Itô-Taylor expansion, it is easy to obtain ( [38])

$$
\left|\int_{t_{n}}^{t_{n+1}}\left(\mathbb{E}_{t_{n}}^{x}\left[f_{s}\right]-\frac{1}{2} f_{t_{n}}-\frac{1}{2} \mathbb{E}_{t_{n}}^{x}\left[f_{t_{n+1}}\right]\right) d s\right| \leq C\left(\Delta t_{n}\right)^{3} .
$$

Since $R_{y 1}^{n}=Y_{t_{n}}-\bar{Y}_{t_{n}}$, then by (2.7) and (3.41a), we deduce

$$
\begin{aligned}
\left|f_{t_{n}, h}-\bar{f}_{t_{n}, h}\right| & \leq C\left(\left|Y_{t_{n}}-\bar{Y}_{t_{n}}\right|+\left|D_{h}^{0} Y_{t_{n}}-D_{h}^{0} \bar{Y}_{t_{n}}\right|\right) \\
& \leq C\left(\left(\Delta t_{n}\right)^{2}+h^{4}\right) .
\end{aligned}
$$

Using (3.44)-(3.47), we get the inequality (3.41b).

For the term $R_{y 3}^{n}=R_{Y 1}^{n}+R_{Y 2}^{n}+R_{Y 3}^{n}$ and $R_{y 4}^{n}=\hat{R}_{Y 1}^{n}+\hat{R}_{Y 2}^{n}+\hat{R}_{Y 3}^{n}$, we have the following theorem.

Theorem 3.2. Under Assumption 3.1, we have the following local estimates for $n=0,1, \cdots, N-1$ :

$$
\begin{aligned}
& \left|R_{y 3}^{n}\right| \leq C\left(\left(\Delta t_{n}\right)^{4}+h^{4} \Delta t_{n}\right), \\
& \left|R_{y 4}^{n}\right| \leq C\left(\left(\Delta t_{n}\right)^{5}+h^{4} \Delta t_{n}\right),
\end{aligned}
$$

where $C$ is a positive constant depending on $T$ and the upper bounds of derivatives of $b, \sigma, f$ and $\varphi$.

Proof. Utilizing (3.16) and (3.27), it is easy to deduce

$$
\left|R_{Y 1}^{n}\right| \leq C\left(\Delta t_{n}\right)^{4}, \quad\left|R_{Y 2}^{n}\right| \leq C\left(h^{4} \Delta t_{n}\right) .
$$

Moreover, $R_{y 2}^{n}=Y_{t_{n}}-\overline{\bar{Y}}_{t_{n}}$, then by (2.7) and (3.41b), we have

$$
\begin{aligned}
& \left|f_{t_{n}, h}-\overline{\bar{f}}_{t_{n}, h}\right| \leq C\left(\left|Y_{t_{n}}-\overline{\bar{Y}}_{t_{n}}\right|+\left|D_{h}^{0} Y_{t_{n}}-D_{h}^{0} \overline{\bar{Y}}_{t_{n}}\right|\right) \leq C\left(\left(\Delta t_{n}\right)^{3}+h^{4}\right), \\
& \left|F_{t_{n}, h}-\overline{\bar{F}}_{t_{n}, h}\right| \leq C\left(\left|Y_{t_{n}}-\overline{\bar{Y}}_{t_{n}}\right|+\left|D_{h}^{0} Y_{t_{n}}-D_{h}^{0} \overline{\bar{Y}}_{t_{n}}\right|+\left|D_{h}^{2} Y_{t_{n}}-D_{h}^{2} \overline{\bar{Y}}_{t_{n}}\right|\right) \leq C\left(\left(\Delta t_{n}\right)^{3}+h^{4}\right),
\end{aligned}
$$

which imply that

$$
\begin{aligned}
\left|R_{Y 3}^{n}\right| & =\left|\theta \Delta t_{n}\left(f_{t_{n}, h}-\overline{\bar{f}}_{t_{n}, h}\right)+\frac{(3 \theta-1)\left(\Delta t_{n}\right)^{2}}{6}\left(F_{t_{n}, h}-\overline{\bar{F}}_{t_{n}, h}\right)\right| \\
& \leq C\left(\left(\Delta t_{n}\right)^{4}+h^{4} \Delta t_{n}\right) .
\end{aligned}
$$

Eqs. (3.49) and (3.50) lead to the inequality (3.48a).

Note that $\hat{R}_{Y 1}^{n}$ and $\hat{R}_{Y 2}^{n}$ are given by taking $\theta=\frac{1}{2}$ in $R_{Y 1}^{n}$ and $R_{Y 2}^{n}$. Then by (3.16) and (3.27), we get

$$
\left|\hat{R}_{Y 1}^{n}\right| \leq C\left(\left(\Delta t_{n}\right)^{5}\right), \quad\left|\hat{R}_{Y 2}^{n}\right| \leq C\left(h^{4} \Delta t_{n}\right) .
$$


By (3.34) and (3.37), we have $R_{y 3}^{n}=Y_{t_{n}}-\hat{Y}_{t_{n}}$. Then by (2.7) and (3.48a), we deduce

$$
\begin{aligned}
& \left|f_{t_{n}, h}-\hat{f}_{t_{n}, h}\right| \leq C\left(\left|Y_{t_{n}}-\hat{Y}_{t_{n}}\right|+\left|D_{h}^{0} Y_{t_{n}}-D_{h}^{0} \hat{Y}_{t_{n}}\right|\right) \leq C\left(\left(\Delta t_{n}\right)^{4}+h^{4}\right), \\
& \left|F_{t_{n}, h}-\hat{F}_{t_{n}, h}\right| \leq C\left(\left|Y_{t_{n}}-\hat{Y}_{t_{n}}\right|+\left|D_{h}^{0} Y_{t_{n}}-D_{h}^{0} \hat{Y}_{t_{n}}\right|+\left|D_{h}^{2} Y_{t_{n}}-D_{h}^{2} \hat{Y}_{t_{n}}\right|\right) \leq C\left(\left(\Delta t_{n}\right)^{4}+h^{4}\right),
\end{aligned}
$$

which imply that

$$
\begin{aligned}
\left|\hat{R}_{Y 3}^{n}\right| & =\left|\frac{1}{2} \Delta t_{n}\left(f_{t_{n}, h}-\hat{f}_{t_{n}, h}\right)+\frac{1}{12}\left(\Delta t_{n}\right)^{2}\left(F_{t_{n}, h}-\hat{F}_{t_{n}, h}\right)\right| \\
& \leq C\left(\left(\Delta t_{n}\right)^{5}+h^{4} \Delta t_{n}\right) .
\end{aligned}
$$

Using (3.51) and (3.52), we obtain the inequality (3.48b).

By Theorem 3.2, we have

$$
\begin{cases}R_{y 3}^{n}=\mathcal{O}\left(\left(\Delta t_{n}\right)^{4}\right), & \text { if } h=\mathcal{O}\left(\left(\Delta t_{n}\right)^{\frac{3}{4}}\right) \\ R_{y 4}^{n}=\mathcal{O}\left(\left(\Delta t_{n}\right)^{5}\right), & \text { if } h=\mathcal{O}\left(\Delta t_{n}\right)\end{cases}
$$

Hence, we can expect that

1. Scheme 3.1 is convergent with third order when we choose $h=\left(\Delta t_{n}\right)^{\frac{3}{4}}$.

2. Scheme 3.2 is convergent with fourth order when we choose $h=\Delta t_{n}$.

\section{Numerical tests}

In this section, we present some numerical experiments to illustrate the stability and accuracy of the proposed schemes. For simplicity, we adopt the uniform time partition, and the time partition number $N$ is given by $N=\frac{T}{\Delta t}$. For some $h>0$, the space partition $\mathcal{S}_{h}$ is chosen as

$$
\mathcal{S}_{h}=\mathcal{S}_{1, h} \times \mathcal{S}_{2, h} \times \cdots \times \mathcal{S}_{d, h}
$$

where $\mathcal{S}_{j, h}$ is the partition of the one-dimensional real axis $\mathbb{R}$

$$
\mathcal{S}_{j, h}=\left\{x_{i}^{j}: x_{i}^{j}=i h, i=0, \pm 1, \cdots, \pm \infty\right\}
$$

for $j=1,2, \cdots, d$. 


\subsection{The approximation of conditional expectation}

By using the distribution of the Brownian motion and the Gauss-Hermite quadrature rule, we show how to approximate the conditional expectation $\mathbb{E}_{t_{n}}^{x}[\cdot]$.

Without loss of generality, we take $\mathbb{E}_{t_{n}}^{x}\left[Y^{n+1}\right]$ for example and use the Euler scheme to solve $X_{t}$. Then we get

$$
\begin{aligned}
\mathbb{E}_{t_{n}}^{X^{n}}\left[Y^{n+1}\right] & =\mathbb{E}_{t_{n}}^{X^{n}}\left[Y^{n+1}\left(X^{n+1}\right)\right] \\
& =\mathbb{E}\left[Y^{n+1}\left(X^{n}+b^{n} \Delta t_{n}+\sigma^{n} \Delta W_{n}\right)\right] \\
& =\frac{1}{\sqrt{2 \pi}} \int_{\mathbb{R}} Y^{n+1}\left(X^{n}+b^{n} \Delta t_{n}+\sigma^{n} \sqrt{\Delta t_{n}} s\right) e^{-\frac{s^{2}}{2}} d s \\
& =\frac{1}{\sqrt{\pi}} \int_{\mathbb{R}} Y^{n+1}\left(X^{n}+b^{n} \Delta t_{n}+\sigma^{n} \sqrt{2 \Delta t_{n}} p\right) e^{-p^{2}} d p \\
& \approx \sum_{i=1}^{M} Y^{n+1}\left(X^{n}+b^{n} \Delta t_{n}+\sigma^{n} \sqrt{2 \Delta t_{n}} a_{i}\right) w_{i},
\end{aligned}
$$

where $\left\{a_{i}\right\}_{i=1}^{M}$ are the roots of the Hermite polynomial of degree $M$ and $\left\{w_{i}\right\}_{i=1}^{M}$ are the corresponding weights [1].

In general,

$$
X_{i}^{n}=X^{n}+b^{n} \Delta t_{n}+\sigma^{n} \sqrt{2 \Delta t_{n}} a_{i}
$$

are not on the grid points. Hence, the interpolation methods are needed. Let $I_{h}$ denote the local interpolation operator such that $I_{h} g(x)=g(x)$ for $x \in S_{h}$, and we obtain

$$
\mathbb{E}_{t_{n}}^{X^{n}}\left[Y^{n+1}\right] \approx \sum_{i=1}^{M} I_{h} Y^{n+1}\left(X^{n}+b^{n} \Delta t_{n}+\sigma^{n} \sqrt{2 \Delta t_{n}} a_{i}\right) w_{i} .
$$

In all our examples, we shall set the number of Gauss-Hermite quadrature points $M=8$ such that the spacial approximated error caused by the Gauss-Hermite quadrature rule can be neglected.

Remark 4.1. Note that Gauss-Hermite quadrature rule and interpolation methods are used to approximate the conditional expectations in our schemes, which will cause the exponential growth in computation when solving high dimensional FBSDEs.

To solve this problem, in our future study, we shall focus on building an efficient sparse grid spatial discretization, using the sparse grid Gaussian-Hermite quadrature rule to approximate the conditional expectations and adopting an efficient spectral method to deal with the associated high dimensional interpolations.

\subsection{Some numerical tests}

In our tests, we set the terminal time $T=1.0$. We also denote by $\left|Y_{0}-Y^{0}\right|$ and $\left|Z_{0}-Z^{0}\right|$ the absolute errors between the exact solution $\left(Y_{t}, Z_{t}\right)$ of the FBSDE (1.1) at time $t=0$ and the 
numerical solutions $\left(Y^{n}, Z^{n}\right)$ of Schemes 3.1 and 3.2 at $n=0$. Moreover, we will choose $h=(\Delta t)^{\frac{3}{4}}$ in Scheme 3.1 and $h=\Delta t$ in Scheme 3.2, respectively.

The convergence rate $(\mathrm{CR})$ with respect to the time step $\Delta t$ is obtained by using linear least square fitting to the numerical errors.

Example 4.1. Consider the following pure BSDE

$$
\left\{\begin{array}{l}
-d Y_{t}=\frac{1}{2}\left(\exp \left(t^{2}\right)-4 t Y_{t}-3 \exp \left(t^{2}-Y_{t} \exp \left(-t^{2}\right)\right)-Z_{t}^{2} \exp \left(-t^{2}\right)\right) d t-Z_{t} d W_{t} \\
Y_{T}=\exp \left(T^{2}\right) \ln \left(3+\sin \left(W_{T}\right)\right)
\end{array}\right.
$$

It can be checked that the analytic solution yields

$$
Y_{t}=\exp \left(t^{2}\right) \ln \left(3+\sin \left(W_{t}\right)\right), \quad Z_{t}=\exp \left(t^{2}\right) \frac{\cos \left(W_{t}\right)}{3+\sin \left(W_{t}\right)}
$$

We use Schemes 3.1 and 3.2 to solve the BSDE (4.3). In Tables 1 and 2, we have listed the numerical errors $\left|Y_{0}-Y^{0}\right|$ and $\left|Z_{0}-Z^{0}\right|$ and the convergence rates of Schemes 3.1 and 3.2 , respectively.

From the numerical results in Tables 1 and 2, we can conclude that Schemes 3.1 and 3.2 are stable and accurate for solving the pure BSDEs for different values of the parameter $\theta$. Moreover, Scheme 3.1 is convergent with order three and Scheme 3.2 with order four, which are consistent with theoretical estimates in Theorem 3.2.

Table 1: Errors and convergence rates of Scheme 3.1 for Example 4.1.

\begin{tabular}{||c|c|c|c|c|c|c||}
\hline & \multicolumn{2}{|c|}{$\theta=0$} & \multicolumn{2}{c|}{$\theta=\frac{1}{4}$} & \multicolumn{2}{c||}{$\theta=\frac{1}{3}$} \\
\hline$N$ & $\left|Y_{0}-Y^{0}\right|$ & $\left|Z_{0}-Z^{0}\right|$ & $\left|Y_{0}-Y^{0}\right|$ & $\left|Z_{0}-Z^{0}\right|$ & $\left|Y_{0}-Y^{0}\right|$ & $\left|Z_{0}-Z^{0}\right|$ \\
\hline 8 & $4.183 \mathrm{E}-03$ & $1.992 \mathrm{E}-04$ & $2.117 \mathrm{E}-03$ & $7.696 \mathrm{E}-05$ & $1.428 \mathrm{E}-03$ & $3.267 \mathrm{E}-05$ \\
16 & $5.079 \mathrm{E}-04$ & $2.126 \mathrm{E}-05$ & $2.534 \mathrm{E}-04$ & $7.879 \mathrm{E}-06$ & $1.685 \mathrm{E}-04$ & $2.770 \mathrm{E}-06$ \\
32 & $6.250 \mathrm{E}-05$ & $2.422 \mathrm{E}-06$ & $3.099 \mathrm{E}-05$ & $9.009 \mathrm{E}-07$ & $2.048 \mathrm{E}-05$ & $2.946 \mathrm{E}-07$ \\
64 & $7.748 \mathrm{E}-06$ & $2.877 \mathrm{E}-07$ & $3.831 \mathrm{E}-06$ & $1.113 \mathrm{E}-07$ & $2.523 \mathrm{E}-06$ & $3.749 \mathrm{E}-08$ \\
128 & $9.646 \mathrm{E}-07$ & $3.505 \mathrm{E}-08$ & $4.765 \mathrm{E}-07$ & $1.441 \mathrm{E}-08$ & $3.135 \mathrm{E}-07$ & $5.315 \mathrm{E}-09$ \\
\hline $\mathrm{CR}$ & 3.020 & 3.115 & 3.028 & 3.091 & 3.037 & 3.138 \\
\hline
\end{tabular}

\begin{tabular}{||c|c|c|c|c|c|c||}
\hline & \multicolumn{2}{|c|}{$\theta=\frac{1}{2}$} & \multicolumn{2}{c|}{$\theta=\frac{2}{3}$} & \multicolumn{2}{c||}{$\theta=1$} \\
\hline$N$ & $\left|Y_{0}-Y^{0}\right|$ & $\left|Z_{0}-Z^{0}\right|$ & $\left|Y_{0}-Y^{0}\right|$ & $\left|Z_{0}-Z^{0}\right|$ & $\left|Y_{0}-Y^{0}\right|$ & $\left|Z_{0}-Z^{0}\right|$ \\
\hline 8 & $5.044 \mathrm{E}-05$ & $6.670 \mathrm{E}-05$ & $1.328 \mathrm{E}-03$ & $1.549 \mathrm{E}-04$ & $4.081 \mathrm{E}-03$ & $3.307 \mathrm{E}-04$ \\
16 & $1.532 \mathrm{E}-06$ & $9.185 \mathrm{E}-06$ & $1.714 \mathrm{E}-04$ & $1.940 \mathrm{E}-05$ & $5.110 \mathrm{E}-04$ & $3.972 \mathrm{E}-05$ \\
32 & $6.027 \mathrm{E}-07$ & $1.207 \mathrm{E}-06$ & $2.164 \mathrm{E}-05$ & $2.418 \mathrm{E}-06$ & $6.371 \mathrm{E}-05$ & $4.837 \mathrm{E}-06$ \\
64 & $9.885 \mathrm{E}-08$ & $1.542 \mathrm{E}-07$ & $2.717 \mathrm{E}-06$ & $3.019 \mathrm{E}-07$ & $7.949 \mathrm{E}-06$ & $5.967 \mathrm{E}-07$ \\
128 & $1.376 \mathrm{E}-08$ & $1.948 \mathrm{E}-08$ & $3.400 \mathrm{E}-07$ & $3.768 \mathrm{E}-08$ & $9.923 \mathrm{E}-07$ & $7.405 \mathrm{E}-08$ \\
\hline CR & 2.763 & 2.938 & 2.984 & 3.002 & 3.002 & 3.031 \\
\hline
\end{tabular}


Table 2: Errors and convergence rates of Scheme 3.2 for Example 4.1.

\begin{tabular}{||c|c|c|c|c|c|c||}
\hline & \multicolumn{2}{|c|}{$\theta=0$} & \multicolumn{2}{c|}{$\theta=\frac{1}{4}$} & \multicolumn{2}{c||}{$\theta=\frac{1}{3}$} \\
\hline$N$ & $\left|Y_{0}-Y^{0}\right|$ & $\left|Z_{0}-Z^{0}\right|$ & $\left|Y_{0}-Y^{0}\right|$ & $\left|Z_{0}-Z^{0}\right|$ & $\left|Y_{0}-Y^{0}\right|$ & $\left|Z_{0}-Z^{0}\right|$ \\
\hline 8 & $1.530 \mathrm{E}-04$ & $3.503 \mathrm{E}-05$ & $4.823 \mathrm{E}-05$ & $1.334 \mathrm{E}-05$ & $1.273 \mathrm{E}-05$ & $2.273 \mathrm{E}-06$ \\
16 & $9.738 \mathrm{E}-06$ & $2.037 \mathrm{E}-06$ & $3.005 \mathrm{E}-06$ & $7.484 \mathrm{E}-07$ & $7.278 \mathrm{E}-07$ & $1.010 \mathrm{E}-07$ \\
32 & $6.102 \mathrm{E}-07$ & $1.218 \mathrm{E}-07$ & $1.860 \mathrm{E}-07$ & $4.362 \mathrm{E}-08$ & $4.272 \mathrm{E}-08$ & $6.662 \mathrm{E}-09$ \\
64 & $3.950 \mathrm{E}-08$ & $7.652 \mathrm{E}-09$ & $1.292 \mathrm{E}-08$ & $2.841 \mathrm{E}-09$ & $3.962 \mathrm{E}-09$ & $5.230 \mathrm{E}-10$ \\
128 & $2.480 \mathrm{E}-09$ & $4.802 \mathrm{E}-10$ & $8.170 \mathrm{E}-10$ & $1.820 \mathrm{E}-10$ & $2.570 \mathrm{E}-10$ & $4.835 \mathrm{E}-11$ \\
\hline $\mathrm{CR}$ & 3.977 & 4.037 & 3.956 & 4.036 & 3.871 & 3.864 \\
\hline
\end{tabular}

\begin{tabular}{||c|c|c|c|c|c|c||}
\hline & \multicolumn{2}{|c|}{$\theta=\frac{1}{2}$} & \multicolumn{2}{c|}{$\theta=\frac{2}{3}$} & \multicolumn{2}{c||}{$\theta=1$} \\
\hline$N$ & $\left|Y_{0}-Y^{0}\right|$ & $\left|Z_{0}-Z^{0}\right|$ & $\left|Y_{0}-Y^{0}\right|$ & $\left|Z_{0}-Z^{0}\right|$ & $\left|Y_{0}-Y^{0}\right|$ & $\left|Z_{0}-Z^{0}\right|$ \\
\hline 8 & $5.653 \mathrm{E}-05$ & $8.353 \mathrm{E}-06$ & $1.264 \mathrm{E}-04$ & $2.282 \mathrm{E}-05$ & $2.660 \mathrm{E}-04$ & $5.174 \mathrm{E}-05$ \\
16 & $3.727 \mathrm{E}-06$ & $5.403 \mathrm{E}-07$ & $8.216 \mathrm{E}-06$ & $1.399 \mathrm{E}-06$ & $1.719 \mathrm{E}-05$ & $3.118 \mathrm{E}-06$ \\
32 & $2.382 \mathrm{E}-07$ & $3.457 \mathrm{E}-08$ & $5.210 \mathrm{E}-07$ & $8.669 \mathrm{E}-08$ & $1.087 \mathrm{E}-06$ & $1.909 \mathrm{E}-07$ \\
64 & $1.366 \mathrm{E}-08$ & $1.969 \mathrm{E}-09$ & $3.138 \mathrm{E}-08$ & $5.176 \mathrm{E}-09$ & $6.683 \mathrm{E}-08$ & $1.159 \mathrm{E}-08$ \\
128 & $8.461 \mathrm{E}-10$ & $1.163 \mathrm{E}-10$ & $1.955 \mathrm{E}-09$ & $3.151 \mathrm{E}-10$ & $4.171 \mathrm{E}-09$ & $7.588 \mathrm{E}-10$ \\
$\mathrm{CR}$ & 4.015 & 4.037 & 3.999 & 4.037 & 3.993 & 4.019 \\
\hline
\end{tabular}

Example 4.2. Consider the following decoupled FBSDE

$$
\left\{\begin{array}{l}
d X_{t}=\sin \left(t+X_{t}\right) d t+\frac{1}{10} \cos \left(t+X_{t}\right) d W_{t}, \\
-d Y_{t}=\left(\frac{1}{20} Y_{t} Z_{t}-\cos \left(t+X_{t}\right)\left(1+\sin \left(t+X_{t}\right)\right)\right) d t-Z_{t} d W_{t},
\end{array}\right.
$$

with the initial value $X_{0}=0$ and the terminal condition $Y_{T}=\sin \left(T+X_{T}\right)$. It can be checked that the analytic solution yields

$$
Y_{t}=\sin \left(t+X_{t}\right), \quad Z_{t}=\frac{1}{10} \cos ^{2}\left(t+X_{t}\right) .
$$

We use the Schemes 3.1 and 3.2 to solve the FBSDE (4.4). For simplicity, we only test our schemes with $\theta=0.5$ and $\theta=1.0$. The Euler scheme (3.1), the Milstein scheme (3.2), the weak order-2.0 Itô-Taylor scheme (3.3), the weak order-3.0 Itô-Taylor scheme (3.4), and the weak order-4.0 Itô-Taylor scheme (3.5) are used to solve SDEs, respectively.

In Tables 3 and 4, we have listed the numerical errors $\left|Y_{0}-Y^{0}\right|$ and $\left|Z_{0}-Z^{0}\right|$ and the convergence rates of Schemes 3.1 and 3.2, respectively.

The errors and the convergence rates in Tables 3 and 4 show that Schemes 3.1 and 3.2 are stable and accurate for solving the decoupled FBSDE (4.4), and their accuracy depends on the Itô-Taylor schemes used for SDEs. From Tables 3 and 4, we can draw the following conclusions.

1. Schemes 3.1 and 3.2 are of order one accurate if the Euler scheme or Milstein scheme are used to solve SDEs. 
Table 3: Errors and convergence rates of Scheme 3.1 for Example 4.2.

\begin{tabular}{||c|c|c|c|c|c|c|c||}
\hline SDE Sch & $N$ & 4 & 8 & 16 & 32 & 64 & CR \\
\hline \multirow{2}{*}{ Euler } & $\left|Y_{0}-Y^{0}\right|$ & $5.624 \mathrm{E}-02$ & $2.825 \mathrm{E}-02$ & $1.422 \mathrm{E}-02$ & $7.527 \mathrm{E}-03$ & $3.697 \mathrm{E}-03$ & 0.976 \\
& $\left|Z_{0}-Z^{0}\right|$ & $3.282 \mathrm{E}-03$ & $8.105 \mathrm{E}-04$ & $8.826 \mathrm{E}-05$ & $2.820 \mathrm{E}-04$ & $1.362 \mathrm{E}-04$ & 1.070 \\
\hline \multirow{2}{*}{ Milstein } & $\left|Y_{0}-Y^{0}\right|$ & $5.624 \mathrm{E}-02$ & $2.785 \mathrm{E}-02$ & $1.486 \mathrm{E}-02$ & $7.457 \mathrm{E}-03$ & $3.828 \mathrm{E}-03$ & 0.965 \\
& $\left|Z_{0}-Z^{0}\right|$ & $3.540 \mathrm{E}-03$ & $1.391 \mathrm{E}-03$ & $4.292 \mathrm{E}-04$ & $2.783 \mathrm{E}-04$ & $1.538 \mathrm{E}-04$ & 1.137 \\
\hline \multirow{2}{*}{ Weak-2.0 } & $\left|Y_{0}-Y^{0}\right|$ & $1.813 \mathrm{E}-03$ & $7.707 \mathrm{E}-04$ & $1.312 \mathrm{E}-04$ & $3.660 \mathrm{E}-05$ & $8.481 \mathrm{E}-06$ & 1.988 \\
& $\left|Z_{0}-Z^{0}\right|$ & $3.468 \mathrm{E}-04$ & $1.034 \mathrm{E}-04$ & $2.452 \mathrm{E}-05$ & $4.029 \mathrm{E}-06$ & $1.078 \mathrm{E}-06$ & 2.134 \\
\hline \multirow{2}{*}{ Weak-3.0 } & $\left|Y_{0}-Y^{0}\right|$ & $2.386 \mathrm{E}-03$ & $2.961 \mathrm{E}-04$ & $3.579 \mathrm{E}-05$ & $4.373 \mathrm{E}-06$ & $5.407 \mathrm{E}-07$ & 3.030 \\
& $\left|Z_{0}-Z^{0}\right|$ & $4.218 \mathrm{E}-05$ & $1.826 \mathrm{E}-06$ & $5.438 \mathrm{E}-07$ & $7.210 \mathrm{E}-08$ & $8.981 \mathrm{E}-09$ & 2.906 \\
\hline \multirow{2}{*}{ Weak-4.0 } & $\left|Y_{0}-Y^{0}\right|$ & $1.001 \mathrm{E}-03$ & $6.047 \mathrm{E}-05$ & $3.954 \mathrm{E}-06$ & $5.424 \mathrm{E}-07$ & $1.689 \mathrm{E}-07$ & 3.187 \\
& $\left|Z_{0}-Z^{0}\right|$ & $1.370 \mathrm{E}-04$ & $1.631 \mathrm{E}-05$ & $1.470 \mathrm{E}-06$ & $1.128 \mathrm{E}-07$ & $6.114 \mathrm{E}-08$ & 2.944 \\
\hline
\end{tabular}

Table 4: Errors and convergence rates of Scheme 3.2 for Example 4.2.

\begin{tabular}{||c|c|c|c|c|c|c|c||}
\hline SDE Sch & $N$ & 4 & 8 & 16 & 32 & 64 & CR \\
\hline \multirow{2}{*}{ Euler } & $\left|Y_{0}-Y^{0}\right|$ & $5.624 \mathrm{E}-02$ & $2.785 \mathrm{E}-02$ & $1.486 \mathrm{E}-02$ & $7.457 \mathrm{E}-03$ & $3.828 \mathrm{E}-03$ & 0.965 \\
& $\left|Z_{0}-Z^{0}\right|$ & $3.541 \mathrm{E}-03$ & $1.391 \mathrm{E}-03$ & $4.292 \mathrm{E}-04$ & $2.783 \mathrm{E}-04$ & $1.538 \mathrm{E}-04$ & 1.137 \\
\hline \multirow{2}{*}{ Milstein } & $\left|Y_{0}-Y^{0}\right|$ & $5.624 \mathrm{E}-02$ & $3.027 \mathrm{E}-02$ & $1.450 \mathrm{E}-02$ & $7.707 \mathrm{E}-03$ & $3.796 \mathrm{E}-03$ & 0.975 \\
& $\left|Z_{0}-Z^{0}\right|$ & $3.781 \mathrm{E}-03$ & $7.372 \mathrm{E}-04$ & $3.148 \mathrm{E}-04$ & $3.194 \mathrm{E}-04$ & $1.469 \mathrm{E}-04$ & 1.058 \\
\hline \multirow{2}{*}{ Weak-2.0 } & $\left|Y_{0}-Y^{0}\right|$ & $1.817 \mathrm{E}-03$ & $7.697 \mathrm{E}-04$ & $1.311 \mathrm{E}-04$ & $3.658 \mathrm{E}-05$ & $8.478 \mathrm{E}-06$ & 1.988 \\
& $\left|Z_{0}-Z^{0}\right|$ & $3.448 \mathrm{E}-04$ & $1.032 \mathrm{E}-04$ & $2.449 \mathrm{E}-05$ & $4.026 \mathrm{E}-06$ & $1.078 \mathrm{E}-06$ & 2.132 \\
\hline \multirow{2}{*}{ Weak-3.0 } & $\left|Y_{0}-Y^{0}\right|$ & $2.391 \mathrm{E}-03$ & $2.971 \mathrm{E}-04$ & $3.595 \mathrm{E}-05$ & $4.394 \mathrm{E}-06$ & $5.433 \mathrm{E}-07$ & 3.029 \\
& $\left|Z_{0}-Z^{0}\right|$ & $4.455 \mathrm{E}-05$ & $1.565 \mathrm{E}-06$ & $5.222 \mathrm{E}-07$ & $7.002 \mathrm{E}-08$ & $8.750 \mathrm{E}-09$ & 2.911 \\
\hline \multirow{2}{*}{ Weak-4.0 } & $\left|Y_{0}-Y^{0}\right|$ & $5.171 \mathrm{E}-04$ & $3.282 \mathrm{E}-05$ & $1.933 \mathrm{E}-06$ & $6.110 \mathrm{E}-09$ & $6.423 \mathrm{E}-08$ & 3.834 \\
& $\left|Z_{0}-Z^{0}\right|$ & $3.984 \mathrm{E}-05$ & $6.188 \mathrm{E}-07$ & $2.153 \mathrm{E}-08$ & $1.863 \mathrm{E}-09$ & $3.602 \mathrm{E}-10$ & 4.189 \\
\hline
\end{tabular}

2. Schemes 3.1 and 3.2 are of order two accurate if the weak order 2.0 Itô-Taylor scheme is used to solve SDEs.

3. Scheme 3.1 is of order three accurate if the weak order 3.0 or weak order 4.0 ItôTaylor schemes are used to solve SDEs.

4. Scheme 3.2 is of order 3.0 accurate if the weak order 3.0 Itô-Taylor scheme is used to solve SDEs.

5. Scheme 3.2 is of order 4.0 accurate if the weak order 4.0 Itô-Taylor scheme is used to solve SDEs.

All of the conclusions above verify again the theoretical estimates in Theorem 3.2. 
Example 4.3. Consider the following decoupled FBSDE

$$
\left\{\begin{array}{l}
d X_{t}=\frac{\exp \left(t+X_{t}\right)}{1+2 \exp \left(t+X_{t}\right)} d t+\frac{\exp \left(t+X_{t}\right)}{10\left(1+\exp \left(t+X_{t}\right)\right)} d W_{t} \\
-d Y_{t}=\left(-\frac{2 Y_{t}}{1+2 \exp \left(t+X_{t}\right)}-\frac{1}{20}\left(\frac{Y_{t} Z_{t}}{1+\exp \left(t+X_{t}\right)}-Y_{t}^{2} Z_{t}\right)\right) d t-Z_{t} d W_{t},
\end{array}\right.
$$

with the initial value $X_{0}=0$ and the terminal condition

$$
Y_{T}=\frac{\exp \left(T+X_{T}\right)}{1+\exp \left(T+X_{T}\right)}
$$

It can be checked that the analytic solution yields

$$
Y_{t}=\frac{\exp \left(t+X_{t}\right)}{1+\exp \left(t+X_{t}\right)}, \quad Z_{t}=\frac{\left(\exp \left(t+X_{t}\right)\right)^{2}}{10\left(1+\exp \left(t+X_{t}\right)\right)^{3}}
$$

We use the Schemes 3.1 and 3.2 to solve the FBSDE (4.5). For simplicity, we test our schemes with $\theta=0.5$ and $\theta=1.0$. The Euler scheme (3.1), the Milstein scheme (3.2), the weak order-2.0 Itô-Taylor scheme (3.3), the weak order-3.0 Itô-Taylor scheme (3.4) and the weak order-4.0 Itô-Taylor scheme (3.5) are used to solve SDEs, respectively.

In Tables 5 and 6 , we have listed the numerical errors $\left|Y_{0}-Y^{0}\right|$ and $\left|Z_{0}-Z^{0}\right|$ and the convergence rates of Schemes 3.1 and 3.2, respectively.

Table 5: Errors and convergence rates of Scheme 3.1 for Example 4.3.

\begin{tabular}{||c|c|c|c|c|c|c|c||} 
SDE Sch & $N$ & 5 & 10 & 15 & 20 & 25 & $C R$ \\
\multirow{2}{*}{ Euler } & $\left|Y_{0}-Y^{0}\right|$ & $3.471 \mathrm{E}-03$ & $1.922 \mathrm{E}-03$ & $1.098 \mathrm{E}-03$ & $8.267 \mathrm{E}-04$ & $7.615 \mathrm{E}-04$ & 0.997 \\
& $\left|Z_{0}-Z^{0}\right|$ & $1.961 \mathrm{E}-04$ & $9.468 \mathrm{E}-05$ & $7.337 \mathrm{E}-05$ & $5.385 \mathrm{E}-05$ & $3.737 \mathrm{E}-05$ & 0.979 \\
\hline \multirow{2}{*}{ Milstein } & $\left|Y_{0}-Y^{0}\right|$ & $3.471 \mathrm{E}-03$ & $1.922 \mathrm{E}-03$ & $1.098 \mathrm{E}-03$ & $8.267 \mathrm{E}-04$ & $7.615 \mathrm{E}-04$ & 0.997 \\
& $\left|Z_{0}-Z^{0}\right|$ & $1.898 \mathrm{E}-04$ & $8.595 \mathrm{E}-05$ & $6.562 \mathrm{E}-05$ & $4.743 \mathrm{E}-05$ & $3.182 \mathrm{E}-05$ & 1.052 \\
& $\left|Y_{0}-Y^{0}\right|$ & $2.122 \mathrm{E}-04$ & $6.237 \mathrm{E}-05$ & $2.352 \mathrm{E}-05$ & $1.324 \mathrm{E}-05$ & $9.616 \mathrm{E}-06$ & 1.977 \\
\multirow{2}{*}{ Weak-2.0 } & $\left|Z_{0}-Z^{0}\right|$ & $2.596 \mathrm{E}-05$ & $8.512 \mathrm{E}-06$ & $3.086 \mathrm{E}-06$ & $1.677 \mathrm{E}-06$ & $1.098 \mathrm{E}-06$ & 2.007 \\
& $\left|Y_{0}-Y^{0}\right|$ & $9.242 \mathrm{E}-06$ & $2.403 \mathrm{E}-06$ & $9.018 \mathrm{E}-07$ & $2.198 \mathrm{E}-08$ & $2.524 \mathrm{E}-07$ & 3.039 \\
\multirow{2}{*}{ Weak-3.0 } & $\left|Z_{0}-Z^{0}\right|$ & $2.644 \mathrm{E}-06$ & $3.444 \mathrm{E}-07$ & $1.172 \mathrm{E}-07$ & $2.188 \mathrm{E}-08$ & $2.891 \mathrm{E}-08$ & 3.034 \\
& $\left|Y_{0}-Y^{0}\right|$ & $6.571 \mathrm{E}-06$ & $2.541 \mathrm{E}-06$ & $9.137 \mathrm{E}-07$ & $4.323 \mathrm{E}-07$ & $1.910 \mathrm{E}-08$ & 3.096 \\
\multirow{2}{*}{ Weak-4.0 } & $\left|Z_{0}-Z^{0}\right|$ & $5.199 \mathrm{E}-06$ & $5.768 \mathrm{E}-07$ & $1.954 \mathrm{E}-07$ & $9.057 \mathrm{E}-08$ & $3.689 \mathrm{E}-08$ & 2.999 \\
\hline
\end{tabular}

The errors and the convergence rates in Tables 5 and 6 imply that Schemes 3.1 and 3.2 are stable and accurate for solving the decoupled FBSDE (4.5), and their accuracy depends on the Taylor schemes used to solve SDEs. Based on the results listed in Tables 5-6, we can draw the same conclusions as the ones in Example 4.2. 
Table 6: Errors and convergence rates of Scheme 3.2 for Example 4.3.

\begin{tabular}{||c|c|c|c|c|c|c|c||}
\hline SDE Sch & $N$ & 5 & 10 & 15 & 20 & 25 & CR \\
\hline \multirow{2}{*}{ Euler } & $\left|Y_{0}-Y^{0}\right|$ & $3.369 \mathrm{E}-03$ & $1.912 \mathrm{E}-03$ & $1.272 \mathrm{E}-03$ & $7.795 \mathrm{E}-04$ & $6.139 \mathrm{E}-04$ & 1.068 \\
& $\left|Z_{0}-Z^{0}\right|$ & $1.945 \mathrm{E}-04$ & $9.129 \mathrm{E}-05$ & $5.758 \mathrm{E}-05$ & $4.925 \mathrm{E}-05$ & $3.957 \mathrm{E}-05$ & 0.990 \\
\hline \multirow{2}{*}{ Milstein } & $\left|Y_{0}-Y^{0}\right|$ & $3.320 \mathrm{E}-03$ & $1.531 \mathrm{E}-03$ & $1.271 \mathrm{E}-03$ & $9.505 \mathrm{E}-04$ & $5.869 \mathrm{E}-04$ & 0.989 \\
& $\left|Z_{0}-Z^{0}\right|$ & $1.963 \mathrm{E}-04$ & $1.078 \mathrm{E}-04$ & $5.719 \mathrm{E}-05$ & $4.297 \mathrm{E}-05$ & $4.079 \mathrm{E}-05$ & 1.047 \\
\hline \multirow{2}{*}{ Weak-2.0 } & $\left|Y_{0}-Y^{0}\right|$ & $2.251 \mathrm{E}-04$ & $6.365 \mathrm{E}-05$ & $2.387 \mathrm{E}-05$ & $1.338 \mathrm{E}-05$ & $9.686 \mathrm{E}-06$ & 2.008 \\
& $\left|Z_{0}-Z^{0}\right|$ & $2.744 \mathrm{E}-05$ & $8.689 \mathrm{E}-06$ & $3.137 \mathrm{E}-06$ & $1.699 \mathrm{E}-06$ & $1.109 \mathrm{E}-06$ & 2.035 \\
\hline \multirow{2}{*}{ Weak-3.0 } & $\left|Y_{0}-Y^{0}\right|$ & $2.065 \mathrm{E}-05$ & $4.193 \mathrm{E}-07$ & $1.519 \mathrm{E}-07$ & $4.054 \mathrm{E}-07$ & $5.311 \mathrm{E}-08$ & 3.241 \\
& $\left|Z_{0}-Z^{0}\right|$ & $6.285 \mathrm{E}-06$ & $3.496 \mathrm{E}-07$ & $1.252 \mathrm{E}-07$ & $7.973 \mathrm{E}-08$ & $3.258 \mathrm{E}-08$ & 3.152 \\
\hline \multirow{2}{*}{ Weak-4.0 } & $\left|Y_{0}-Y^{0}\right|$ & $1.268 \mathrm{E}-05$ & $7.862 \mathrm{E}-07$ & $1.696 \mathrm{E}-07$ & $6.179 \mathrm{E}-08$ & $3.008 \mathrm{E}-08$ & 3.774 \\
& $\left|Z_{0}-Z^{0}\right|$ & $1.036 \mathrm{E}-06$ & $5.486 \mathrm{E}-08$ & $1.165 \mathrm{E}-08$ & $3.629 \mathrm{E}-09$ & $2.227 \mathrm{E}-09$ & 3.891 \\
\hline
\end{tabular}

\section{Conclusions}

In this work, we developed an explicit third order one-step scheme and an explicit fourth order one-step scheme for decoupled FBSDEs. Numerical tests show that the one-step schemes are efficient and are of high order rates of convergence. In our future work, we shall consider to combine the sparse grid method to deal with high dimensional coupled FBSDEs.

\section{Acknowledgements}

The authors would like to thank the referees for their valuable comments and suggestions which helped to improve this paper. This research is partially supported by the NSF of China (No. 12001539), the NSF of Hunan Province (No. 2020JJ5647) and China Postdoctoral Science Foundation (No. 2019TQ0073).

\section{References}

[1] M. Abramowitz AND I. STEgun, Handbook of Mathematical Functions, Dover, New York, 1972.

[2] R. Buckdahn, B. Djehiche, J. Li And S. Peng, Mean-field backward stochastic differential equations: a limit approach, Ann. Probab., 37 (2009), pp. 1524-1565.

[3] R. BucKdahn, J. Li AND S. Peng, Mean-field backward stochastic differential equations and related partial differential equations, Stochastic Process. Appl., 119 (2009), pp. 3133-3154.

[4] B. BOUCHARD AND N. TouzI, Discrete-time approximation and Monte-Carlo simulation of backward stochastic differential equations, Stochastic Process. Appl., 111 (2004), pp. 175-206.

[5] D. ChevanCE, Numerical methods for backward stochastic differential equations, J. Optim. Theory Appl., 47 (1997), pp. 1-16.

[6] F. Delarue AND S. MeNOZZI, An interpolated stochastic algorithm for quasi-linear pdes, Math. Comput., 77 (2008), pp. 125-158 
[7] J. Douglas, J. MA AND P. PROTTER, Numerical methods for forward-backward stochastic differential equations, Ann. Appl. Prob., 6 (1996), pp. 940-968.

[8] Y. FU AND W. ZHAO, An explicit second-order numerical scheme to solve decoupled forward backward stochastic differential equations, East Asian J. Appl. Math., 4 (2014), pp. 368-385.

[9] Y. FU, W. ZHAO AND T. ZHOU, Multistep schemes for forward backward stochastic differential equations with jumps, J. Sci. Comput., 69 (2016), pp. 651-672.

[10] Y. FU, W. ZHAO AND T. ZHOU, Efficient spectral sparse grid approximations for solving multidimensional forward backward SDEs, Discrete Contin. Dyn. Syst. Ser. B, 22 (2017), pp. 34393458.

[11] E. R. GIANIN, Risk measures via g-expectations, Insurance Math. Econom., 39 (2006), pp. 1934.

[12] E. GOBET AND C. LABART, Error expansion for the discretization of backward stochastic differential equations, Stochastic Process. Appl., 117 (2007), pp. 803-829.

[13] E. Gobet, J. P. Lemor AND X. WARIN, A regression-based Monte Carlo method to solve backward stochastic differential equations, Ann. Appl. Probab., 3 (2005), pp. 2172-2202.

[14] S. HAMADENE AND J. P. LEPELTIER, Zero-sum stochastic differential games and backward equations, Systems Control Lett., 24 (1995), pp. 259-263.

[15] N. E. Karoui, S. Peng AND M. C. QueneZ, Backward stochastic differential equations in finance, Math. Finance, 7 (1997), pp. 1-71.

[16] P. Kloeden And P. Platen, Numerical Solution of Stochastic Differential Equations, Springer-Verlag, Berlin, 1992.

[17] T. KONG, W. ZHAO AND T. ZHOU, Probabilistic high order numerical schemes for fully nonlinear parabolic PDEs, Commun. Comput. Phys., 18 (2015), pp. 1482-1503.

[18] A. LIONNET, D. R. GONCALO AND S. LUKASZ, Time discretization of FBSDE with polynomial growth drivers and reaction-diffusion PDEs, Ann. Appl. Probab., 5 (2015), pp. 2563-2625.

[19] Y. LI, J. YANG AND W. ZHAO, Convergence error estimates of the Crank-Nicolson scheme for solving decoupled FBSDEs, Sci. China Math., 60 (2017), pp. 923-948.

[20] J. MA, P. PROTTER AND J. YONG, Solving forward-backward stochastic differential equations explicitly-a four step scheme, Probab. Theory Related Fields, 98 (1994), pp. 339-359.

[21] G. N. MilsteIN AND M. V. TRETYAKOV, Discretization of forward-backward stochastic differential equations and related quasi-linear parabolic equations, SIAM J. Numer. Anal., 27 (2007), pp. 24-44.

[22] E. Pardoux And S. Peng, Adapted solution of a backward stochastic differential equation, Systems Control Lett., 14 (1990), pp. 55-61.

[23] S. PENG, A general stochastic maximum principle for optimal control problems, SIAM J. Control Optim., 28 (1990), pp. 966-979.

[24] S. PENG, Probabilistic interpretation for systems of quasilinear parabolic partial differential equations, Stochastics Stochastics Rep., 37 (1991), pp. 61-74.

[25] Y. SUN AND W. ZHAO, New second-order schemes for forward backward stochastic differential equations, East Asian J. Appl. Math., 8 (2018), pp. 399-421.

[26] Y. SUN AND W. ZHAO, An explicit second-order numerical scheme for mean-field forward backward stochastic differential equations, Numer. Algorithms, 84 (2020), pp. 253-283.

[27] Y. SUN, J. YANG AND W. ZHAO, Itô-Taylor schemes for solving mean-field stochastic differential equations, Numer. Math. Theor. Meth. Appl., 10 (2017), pp. 798-828.

[28] Y. SUN, W. ZHAO AND T. ZHOU, Explicit $\theta$-scheme for solving mean-field backward stochastic differential equations, SIAM J. Numer. Anal., 56 (2018), pp. 2672-2697.

[29] T. TANG, W. ZHAO AND Z. ZHOU, Deferred correction methods for forward backward stochastic 
differential equations, Numer. Math. Theor. Meth. Appl., 10 (2017), pp. 222-242.

[30] J. YANG AND W. ZHAO, Convergence of recent multistep schemes for a forward-backward stochastic differential equation, East Asian J. Appl. Math., 5 (2015), pp. 387-404.

[31] C. ZHANG, J. WU AND W. ZHAO, One-step multi-derivative methods for backward stochastic differential equations, Numer. Math. Theor. Meth. Appl., 12 (2019), pp. 1213-1230.

[32] W. ZhaO, L. Chen AND S. Peng, A new kind of accurate numerical method for backward stochastic differential equations, SIAM J. Sci. Comput., 28 (2006), pp. 1563-1581.

[33] W. ZHAO, J. WANG AND S. PENG, Error estimates of the $\theta$-scheme for backward stochastic differential equations, Discrete Contin. Dyn. Syst. Ser. B, 12 (2009), pp. 905-924.

[34] W. ZHAO, Y. FU AND T. ZHOU, New kinds of high-order multistep schemes for coupled forward backward stochastic differential equations, SIAM J. Sci. Comput., 36 (2014), pp. A1731-A1751.

[35] W. ZHAO, Y. LI AND Y. FU, Second-order schemes for solving decoupled forward backward stochastic differential equations, Sci. China Math., 57 (2014), pp. 665-686.

[36] W. ZHAO, Y. Li AND G. ZHANG, A generalized $\theta$-scheme for solving backward stochastic differential equations, Discrete Contin. Dyn. Syst. Ser. B, 17 (2012), pp. 1585-1603.

[37] W. ZHAO, G. ZHANG AND L. JU, A stable multistep scheme for solving backward stochastic differential equations, SIAM J. Numer. Anal., 48 (2010), pp. 1369-1394.

[38] W. ZHAO, W. ZHANG AND L. JU, A numerical method and its error estimates for the decoupled forward-backward stochastic differential equations, Commun. Comput. Phys., 15 (2014), pp. 618646.

[39] W. ZHAO, W. ZHANG AND L. JU, A multistep scheme for decoupled forward-backward stochastic differential equations, Numer. Math. Theor. Meth. Appl., 9 (2016), pp. 262-288. 